\title{
PERMEABILITY REDUCTION PHENOMENA IN PACKED BEDS, FIBER MATS, AND WET WEBS OF PAPER EXPOSED TO FLOW OF LIQUIDS AND SUSPENSIONS: A REVIEW
}

\author{
Martin A. Hubbe, ${ }^{*}$ Hao Chen, and John A. Heitmann \\ Filter media, including those prepared from cellulosic materials, often \\ suffer from permeability loss during continued use. To help understand \\ such issues, one can take advantage of an extensive body of related \\ research in such fields as industrial filtration, water purification, \\ enhanced oil recovery, chromatography, paper manufacture, and the \\ leaching of pollutants from impoundments. Though the mechanisms that \\ appear to govern permeability-loss phenomena depend a lot on the \\ details of various applications, the published research has revealed a \\ number of common features. In particular, flow through a porous bed or \\ fiber mat can be markedly reduced by deposition of particles or colloidal \\ matter in positions that either block or partially restrict fluid flow. \\ Progress has been achieved in the development of mechanistic models, \\ as well as the use of such models in numerical simulations to explain \\ various experimental findings. Further research of this type needs to be \\ applied to cellulosic materials, which tend to be much more elongated in \\ comparison to the bed materials and suspended matter considered most \\ often by most researchers active in research related to permeability loss.
}

Keywords: Permeability reduction; Flux decline; Clogging; Blocking; Plugging; Sealing; Fouling;

Formation damage; Packed beds; Filtration; Fiber mats; Dewatering of paper

Contact information: Department of Forest Biomaterials, North Carolina State University, Campus Box 8005; Raleigh, NC 27695-8005; *Corresponding author: hubbe@ncsu.edu

\section{INTRODUCTION}

Instances of clogging, fouling, or progressive losses in permeability of filter media, packed beds, and fibrous mats have been reported in disparate fields of application. Numerous publications have dealt with this issue as it affects filtration operations, the recovery of petroleum, the leaching of pollutants in soils, and in many other applications to be mentioned in the course of this review article. In terms of cellulosic materials the subject can be critically important during the formation and dewatering of paper, and also when cellulosic materials are being used as filter media. However, as will be noted at the end of this review, cellulosic materials tend to be much more elongated in comparison to other materials that have been included in most studies involving filter media, suspended matter, and how the solids affect permeability reduction phenomena. Nevertheless, some of the published approaches to understanding of permeability reduction have potential to either be applied in an approximate sense or modified to be able to better explain permeability reduction phenomena associated with cellulosic materials. 
Porous materials can differ greatly with respect not only to pore size, but also in terms of morphology and the degree to which pores are connected to one another (Ishizaki et al. 1998). In studies of various filter media it is common to identify certain limiting situations. So-called “deep-bed filtration” removes particles from a liquid suspension as it passes through the filter bed; this type of separation is most often carried out with granular material, such as sand. Membranes are at the other extreme, since a typical membrane filter is thin enough that individual pores can extend from one side to the other of the medium. The term "cake filtration" or "surface filtration" is often used to describe situations in which solids from the filtered stream tend to build up on the surface of a membrane or a screen, causing particle removal to occur in the accumulated cake material prior to reaching the membrane or screen.

Papermaking also can be regarded as its own unique type of filtration operation. The paper forming process resembles cake filtration insofar as the material accumulates on top of a more highly permeable barrier. But papermaking differs from ordinary cake filtration due to the fact that both the fine matter and the fibers arrive simultaneously at the site of mat buildup. The openings in the forming fabric are too large to mechanically retain the fines fraction; thus the fiber mat itself, as it is being formed and densified, begins to play a more and more effective role in retaining the finer particles. Papermaking also can be thought of as a kind of deep bed filtration, as long as one bears in mind that the filter medium is really not very "deep," that the void volume of the paper web decreases during the process, and that the mat is fibrous, rather than the more widely studied granular type of filter bed. In addition, the dewatering of a paper web during commercial papermaking does not involve steady, laminar flow. Rather, a pulsating pattern of vacuum applications, often at gradually increasing intensity, are designed to promote more rapid release of water (Hubbe and Heitmann 2007). Papermakers share a key pair of goals with their counterparts in the filtration industry, that is, to collect fine particles while at the same time avoiding excessive loss in permeability of the filter medium.

\section{Fields of Application}

Filtration

An early systematic analysis of permeability loss during filtration involved the purification of drinking water. Iwasaki (1937) reported a gradual reduction in flux through a sand filter bed and proposed that the loss in permeability was associated with the filling of void spaces within the sand. Even a small amount of bacteria present in the water to be purified was sufficient to greatly increase the time required for filtration. Sand filtration has continued to be of great interest to many researchers (Fan et al. 1985; Darby and Lawler 1990; Tobiason and Vigneswaran 1994; Elimelech et al. 1995; Rijnaarts et al. 1996; Kuhnen et al. 2000; Amburgey and Amirtharajah 2005; Broadford et al. 2005; Raveendran and Amirtharajah 1995; Zhao et al. 2005; Nascimento et al. 2006).

Sand filtration falls within a larger class of industrial operations called "granular deep-bed filtration” (Tien and Ramarao 2008). Experimental studies of deep-bed filtration have been widely reported (Grace 1956; Heertjes 1957; Maroudas and Eisenklam 1965a; O’Melia and Ali 1978; Pendse et al. 1978; Johnson and Elimelech 
1995; Putnam and Burns 1997; Iliuta and larachi 2003, 2005, 2006; Jegatheesan et al. 2005; Yoon et al. 2006). Due to the extensive and diverse applications of deep-bed filtration, there have been many attempts to fit empirical and theoretical equations to pressure loss data (Hermans and Bredée 1936; Ives and Pienvichitr 1965; Maroudas and Eisenklam 1965b; Herzig et al. 1970; Tien et al. 1979; Payatakes et al. 1973; 1981; Chiang and Tien 1985; Fan et al. 1985a,b; McDowell-Boyer 1986; Mackie et al. 1987; Sharma and Yortsos 1987a-c; Rege and Fogler 1988; Tobiason and Vigneswaran 1994; Boller and Kavanaugh 1995; Bai and Tien 1997; Narayan et al. 1997; Pandya et al. 1998; Data and Redner 1998; Le Coq and Silvy 1999; Lee and Koplik 2001; Jegatheesan et al. 2005; Mays and Hunt 2005; Shapiro et al. 2005; Zhao et al. 2005).

When it comes to water filtration, considerable attention has shifted to the use of membranes. In principle, membrane filtration can be designed to exclude bacteria from the filtered water. Although membrane filtration differs from deep-bed filtration in many ways, the observed reductions in permeability can be similar (Davis 1992; Matsumoto et al. 1992; McDonough et al. 1992; Belfort et al. 1993; Bowen et al. 1995; Iritani et al. 1995, 2007; Murase et al. 1995; Ho and Zydney 2000; Skerlos et al. 2001; Boerlage et al. 2002; Howe and Clark 2002; Seminario et al. 2002; Jiang et al. 2003; Zhang et al. 2003; Tarabara et al. 2004; Zhao et al. 2004; De Bruin et al. 2005; Kilduff et al. 2005; Meng et al. 2005; Purkait et al. 2005; Singh and Song 2005; Bolton et al. 2005, 2006; DuclosOrsello et al. 2006; Gironès et al. 2006; Mourouzidis-Mourouzis and Karabelas 2006; Katsoufidou et al. 2008).

\section{Movement of toxins in groundwater}

Permeability changes also can play a significant role in the movement of groundwater through soils and within the water table (Bradford et al. 2004). Researchers have shown particular interest in mechanisms involving the transport of heavy metal ions and other toxins that happen to be adsorbed on colloidal particles that are transported by the groundwater (Ryan and Elimelech 1996; Kuhnen et al. 2000; Simunek et al. 2006). Because such particles may become entrapped at constrictions within the pore structure, one can expect that mechanisms related to permeation can be important.

\section{Sealing of waste lagoons}

Permeability reductions can be highly desirable when it comes to the sealing of wastewater lagoons, waste landfills, and similar impoundments. Montmorillonite clay, sometimes called "bentonite," has been used for such applications (Curry and Beasley 1962). Goldenberg (1985) showed that very small amounts of well-dispersed montmorillonite clay were able to decrease the permeability of sand beds by factors of 100 to 1000 . Rausch and Curry (1963) showed that reduced permeability could be maximized by controlling the state of aggregation of montmorillonite clay. Tyner et al. (2006) observed that particles present in dairy waste were more important than the underlying soil with respect to the sealing of a waste lagoon.

\section{Petroleum: formation damage}

Permeability issues are of great concern to the petroleum industry. Many researchers have reported that inappropriate composition of injected water during 
enhanced oil recovery can cause substantial losses in flow through the formation (Muecke 1979; Reed 1980; Gruesbeck and Collins 1982; Kruger 1986; Sharma et al. 1987; Bedrikovetsky et al. 2001; Al-Abduwai et al. 2003; Alvarez 2007; Mojarad and Settari 2007). The phenomenon is known in the industry as "formation damage."

A surprising observation, which has been confirmed in many studies related to oilfield applications, is that severe losses in permeability can occur even in cases where the injected fluid is completely free of particulates. Rather, blockage of pores in the geologic substrata often occurs when the injected fluid has a much lower salt content in comparison to the water initially present (Johnston and Beeson 1945; Monaghan et al. 1959; Mungan 1965; Khilar et al. 1983; Kruger 1986; Cerda 1987a,b; Khilar and Fogler 1998). Such effects have been attributed to the release, or "peptization," of fine clay particles from the void spaces of sandstone and related strata. An abrupt change from a salty condition to a fresh water condition can increase the electrostatic repulsive forces between solids, causing fine particles to become released into the solution. Such released particles then are free to follow the flow to locations where they get stuck and tend to impede further flow.

\section{Chromatography}

A characteristic feature of many chromatographic applications is the presence of two or more classes of pore size. For instance, commonly available chromatographic silica gel packing material can have internal pores of 3-50 nm, whereas the passages around the outsides of such particles may be more than 1000 times larger. Larson et al. (2003) and Sin et al. (2006) were among the few researchers who have discussed plugging phenomena and how they affect chromatographic measurements.

\section{Blood flow and pressure}

Though the flow of blood differs in many respects from other applications considered in this article, there are some common features. Research has shown that the blood recirculation system can be subject to two kinds of resistance to flow (Ganter et al. 2006). On the one hand, the blood contents have to pass through fine capillaries as they pass from the arteries to the veins. But the pressure available during those transitions can also be reduced if materials have accumulated on arterial walls, or if other factors have caused the arteries to become constricted.

\section{Papermaking}

The paper forming process can be described as a filtration of mixed suspensions containing cellulosic fibers along with smaller matter, called "fines." Various studies have shown that the release of water from the paper matrix during papermaking could occur much more rapidly, except for the presence of the fines (Britt et al. 1986; Patel and Trivedi 1994; Kumar et al. 1996; Räisänen 1996; Hubbe 2002; Paradis et al. 2002; Wildfong et al. 2000a,b, 2003, Cole et al. 2008; Hubbe et al. 2008). Reductions in rates of dewatering tend to become more important with increasing weight per unit area of paper and with increasing fines content. Related applications were the subject of an earlier review article in this journal (Hubbe and Heitmann 2007). 


\section{Organization of Article}

The next section will consider qualitative descriptions of permeability loss phenomena. Then there will be a section devoted to mathematical descriptions of permeation loss, followed by a section devoted to computer simulation studies. The article will finish up with relatively brief sections devoted to strategies for maintaining the permeability of porous materials, strategies for intentional sealing of permeable materials, and an overall summary.

\section{FACTORS AND EFFECTS}

Effects of various factors will be considered in this section across a broad range of experimental approaches, emphasizing qualitative issues. It will be assumed, for the sake of this discussion, that many of the qualitative findings in published studies have the potential to cast light on permeability loss issues in cellulosic filter media and in papermaking applications.

\section{Flow, Net Solids Injected, and Concentration}

Almost without exception, published articles related to permeability loss have shown a cumulative effect of flow. The more fluid that has passed through the medium, the greater the reduction in permeability. The following references can be considered as representative for this type of finding (McDowell-Boyer et al. 1986; Le Coq and Silvy 1999; Mays and Hunt 2005).

Many studies, however, have shown cases in which the key variable may not be the volume of flow itself, but rather, what is the cumulative amount of filterable material contained in the inlet mixture (Mays and Hunt 2005). For example, Sin et al. (2006) observed no change in permeability when a packed column was exposed to particle-free solution. Data compiled by Mays and Hunt (2005) showed that the deposition of solids equivalent to about $1 \%$ of the filter volume was sufficient to decrease permeability by a factor of 100 to 1000 (see also Goldenberg 1985). It would be tempting to conclude that there ought to be a simple relationship between the amount of solids to which a filter medium has been exposed and its loss in permeability. However, as shown in the cited data collected by Sin et al. (2006), more severe permeability loss sometimes comes as a result of a more concentrated injection of a particle suspension, in comparison to the same net amount of material over a long time, especially if small injections are separated by rinsing cycles. Likewise, Pandya et al. (1998) found that permeability of a packed bed depended on the concentration of the suspension, in addition to other factors.

\section{Particle Size}

If at least some of the particles in the inflow to a filter medium are larger than the minimum size of pores, then it is easy to understand why particle size often can have a major effect on both collection efficiency and on permeability. Based on such thinking, it would not be surprising that many studies have predicted or shown particle size to be a major factor with respect to loss of permeability, with the larger particles being more 
likely to clog pores (Rege and Fogler 1987; Pandya et al. 1998; Seminario et al. 2002; Bradford et al. 2004).

In practice, smaller particles often produce more reduction in permeability, especially if one considers the effects per unit mass of solids in the infow (O'Melia and Ali 1978; Darby and Lawler 1990; Tobiason and Vigneswaran 1994). As noted in an earlier review (Hubbe and Heitmann 2007), studies have shown greater permeability reductions due to the entrapment of smaller cellulosic materials during the filtration through fiber mats (Przybysz and Szwarcsztajn 1973; Patel et al 1994; Liu et al. 2001). Such results can be explained in terms of fluid frictional effects, which can be expected to be roughly proportional to the effective surface area over which the fluids must pass. The smaller the size of the primary particles, the larger will be the surface area per unit mass.

For many years it has been known that the presence of clays, such as montmorillonite, can greatly reduce the permeability of sandstone and related porous beds (Monaghan et al. 1959; Jones 1964; Mungan 1965; Muecke 1979; Khilar and Fogler 1983; Goldenberg 1985; Kruger 1986; Cerda 1987b). In particular, clogging of the media has been observed when a salty solution is replaced by relatively salt-free water (Khilar and Fogler 1983; Kruger 1986). A possible way to explain such effects is that the montmorillonite swells when the ionic strength of the adjacent solution is decreased (Mohan and Fogler 1997; Hamdi and Srasra 2008; Jimenez-Gonzalez 2008). By swelling, a particle of clay either can occupy more space, or also it may release very thin mineral platelets, which would seem to be ideally suited for the job of blocking flow. Whether or not the mechanism described takes place, there is evidence that considerable reduction in permeability can occur even without such effects. Thus, Mungan (1965) achieved similar effects when non-swelling clays were added to the inlet flow through sandstone samples.

$\mathrm{Fu}$ and Dempsey (1998) predicted a complex relationship between particle size and the permeability of filter cakes, depending on the interactive forces between surfaces. Surface chemical conditions can affect how particles agglomerate and how they deposit onto surfaces, and such interactions also depend on particle shape. Thus, the surface chemical conditions giving the greatest resistance to flow through a filter cake can be expected to be different for different sizes of particles. Surface-chemical effects will be considered in more detail later.

Mechanisms related to particle retention within filter media imply different effects related to particle size. Fan et al. (1985a,b) concluded that the mechanism of particle retention was quite different, depending on the size ranges of particles; large particles were retained mainly by physical factors (straining), whereas the retention of smaller particles appeared to depend more on attractive forces between surfaces. Such predictions are based on trajectory theory (see Yao et al. 1971). Analyses using trajectory theory are based on movements along stream-lines to predict the likelihood that a suspended particle will come into contact with a fixed solid surface. Particle removal requires, however, that not only does the suspended particle collide with a surface, but also that it sticks upon collision. These issues will be considered in a later section dealing with chemical effects. Straining is not considered to be a main mechanism of removal in a typical water treatment operation due to the fact that larger particles tend to settle out of the water by gravity clarification before reaching the filtration operation. 
To illustrate some effects of particle size, consider the two cases compared in Fig. 1. Part A of the figure illustrates how a non-adhesive particle (even if there are repulsive forces between the particle and the pore walls) is expected to become entrapped when it encounters part of the pore network that is too narrow for it to pass. By contrast, mechanical entrapment of smaller particles (see Fig. 1B) might require a complicated bridging mechanism, involving several particles arriving simultaneously at the site of fouling. This mechanism could help explain various effects of particle concentration on filter performance, especially if a highly concentrated particle suspension causes greater blockage in comparison to the same net amount of particles passing through a filter at higher dilution (Khilar et al. 1983; Pandya et al. 1998; Sin et al. 2006). A multi-particle blockage, as in part B, would tend to be somewhat porous, and the suspending medium would be able to flow through it. Although many researchers have proposed "total blockage" of pores, as an idealized mechanism, it is reasonable to expect that even in the case represented in part A of the figure, there would be some leakage of filtrate past the blocking particle.

Another effect of particle size concerns momentum. In general, inertial effects tend to be relatively small for typical filtration operations involving aqueous media, and inertial effects are often neglected for the sake of simplifying the analysis of particle trajectories. However, larger particles have a greater tendency to deviate from curved streamlines of flow due to inertia. Based on this tendency it would be reasonable to expect preferential buildup of larger-particle deposits at the outer side of the curve where the fluid has to bend as it proceeds through a filter medium. As considered later in this article, hydrodynamic forces of detachment also may play a role in such cases.

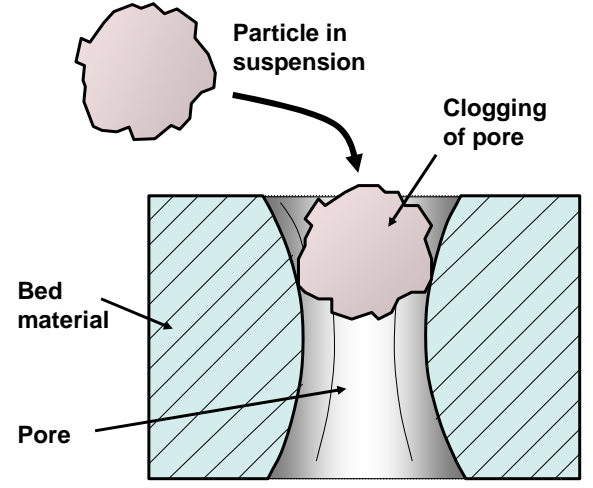

Fig. 1A. Mechanism of particle entrapment by mechanical straining, leading to a complete or partial blockage of one element in a porous network

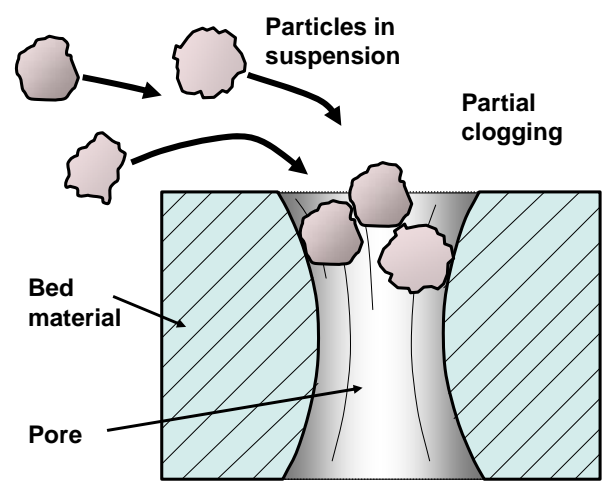

Fig. 1B. Hypothetical blockage of a pore by simultaneous bridging with smaller particles, an event that may be unlikely in the absence of net attractive forces between surfaces

\section{Particle Shape}

Figure 2 represents three kinds of particle shapes that are of potential interest to researchers for filtration operations and for research related to permeability loss. As described in the previous section, clay-like particles have been noted for their tendency to clog filter media. A common explanation for this behavior is that clay-like materials have a platy shape. Depending on its orientation, a platy particle can block a rounded pore constriction very effectively, achieving the blockage with a minimum of mass. 
A

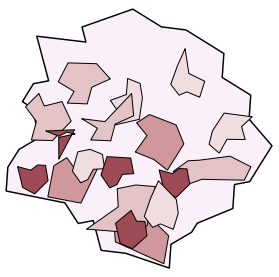

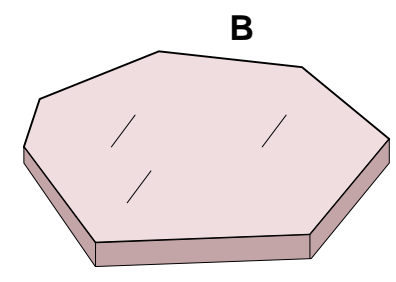

Fig. 2. Characteristic shapes of suspended particles of interest to scientists and technologists: A - granular; B - platey; C - fibrous

Though various authors have experimented with the effects of spherical particle suspensions flowing through filter beds (Ives and Pienvichitr 1965; Darby and Lawler 1990; Duclos-Orsello and Ho 2006), a search of the literature did not reveal any published research systematically comparing the permeation reduction effects of such particles relative to other shapes, such as platy particles or fibrous particles. A rare study dealing with the shapes of particles in suspension was reported by Maroudas and Eisenklam (1965a). These authors found that the clogging tendency of rough, anglular particles can be quite different from that of smooth particles. Smooth particles were observed to gradually build up and constrict pore openings in a packed bed composed of glass spheres. By contrast, the angular particles rapidly blocked passages in the packed bed. These differences were interpreted based on the contrasting morphology of deposits formed from smooth vs. angular particles (Maroudas and Eisenklam 1965b). In addition, it is reasonable to expect smooth, rounded particles to roll more easily away from their sites of attachment.

Effects related to the elongated (i.e. fibrillar or acicular) shapes of suspended particles appear hardly to have been mentioned in the literature, with the exception of studies related to papermaking applications. The following studies all showed that fibrillar cellulosic fines yielded much greater decreases in the permeability of fiber mats in comparison to fines having relatively compact shapes (Brecht and Klemm 1953; Steenberg et al. 1960; Waterhouse and Omori 1993; Krogerus et al. 2002; Hubbe 2002; Cole et al. 2008). It is reasonable to expect that an acicular particle will be much more effective in bridging openings within a packed bed, in comparison to spherical or blocky particles having the same mass.

\section{Bed Properties and Structure}

Streamlines of fluid flowing within a porous medium are forced to take longer paths in comparison to the same fluids flowing through a larger, open channel. The average lengths of such paths depend greatly on the structure of the medium. Tortuosity can be defined in different ways, using the symbols $\tau_{\mathrm{G}}, \tau_{\mathrm{D}}$, and $\tau_{\mathrm{P}}$. The geometric tortuosity $\tau_{\mathrm{G}}$ can be defined as the ratio between the length of the centerline of a typical continuous pore to the straight-line distance through the medium. The diffusion tortuosity $\tau_{\mathrm{D}}$ can be defined as the ratio of diffusivity of a tracer molecule in the bulk solution, in comparison to its ability to diffuse through the porous medium. The quantity $\tau_{\mathrm{D}}$ can be useful for analysis of such properties as thermal conductivity, electrical conductance, and diffusion of solutes, etc. The dynamic tortuosity $\tau_{\mathrm{P}}$ is related to the 
equations of flow and the prediction of flow resistance, usually under the assumption that inertial effects can be neglected. Sakthivadivel et al. (1972) defined tortuosity as the characteristic or average ratio by which the path length has increased in comparison to bulk laminar flow. In qualitative terms, an increase in tortuosity can be expected to increase resistance to flow, since the fluid is forced to travel farther and faster in order to match the same volumetric output. One way to estimate the diffusion tortuosity $\tau_{\mathrm{D}}$ is to measure the electrical conductivity of a bed saturated with a liquid of known conductivity (Wong et al. 2004; Barrande et al. 2007). Assuming that the solids are non-conductive and that the surface conductance can be neglected, tortuosity can be defined as the ratio between the conductivity of the liquid and that of the bed filled with the same liquid. As shown by $\mathrm{Wu}$ et al. (2006), a geometric tortuosity factor $\tau_{\mathrm{G}}$ sometimes can be estimated based on image analysis of different cross-sections taken from a bed. Le Coq (2008) showed that tortuosity factors also can be estimated from detailed structural analysis, using methods such as mercury porosimetry.

Flowing liquid tends to follow the paths of least resistance. This implies that nonuniformities in porous media can have relatively large effects on overall permeability. For instance, Bradford et al. (2004) found that flow tended to avoid fine-grained regions within a sand bed. Carroll (2001) likewise found that nonuniform filter cake permeability was important during certain hollow-fiber microfiltration practices. Dullien (1975) showed that such effects can be dealt with mathematically by assuming a simpler model, (e.g. two different characteristic pore sizes) in order to model the flow behavior through a real system.

The packing density of granular material often depends on the size distribution of the component particles. Small particles of suitable size and amount can fill in the void spaces among larger-sized particles. As a result, a somewhat polydisperse mixture of spheres will produce a denser and less permeable bed in comparison to a bed of equalsized spheres (Alince and Lepoutre 1983; Thies-Weesie and Philipse 1994).

Fractal concepts can permit elegant descriptions related to the structure of porous media. For instance, one can express bed morphology in terms of a dimensionless correlation length (Ioannides and Chatzis 2000). One defines the effective range of repeating features within a packed bed, for instance, by how many grain-equivalent lengths does one have to move within the bed before the position of a reference grain no longer has a statistical effect on whether another space in the medium is occupied by solid or void. Meng et al. (2005) used a related approach to understand the fouling of a membrane in a bioreactor. Though some have questioned the practical utility of fractal analysis for predicting average permeation behavior (Wiesner 1999), one can expect there to be active research in this area in years ahead.

\section{Types of sites}

One of the potential problems with concepts such as tortuosity and fractal dimensions is that they don't consider effects due to different kinds of sites within a porous structure. Thus, Herzig et al. (1970) distinguished between surface sites, crevice sites, constrictions sites, and cavern sites. Both the probability of particle deposition at such sites and the consequent effects on porosity can be expected to be different. Yoon et 
al. (2006) showed that the deposition patterns of particles within a bed of smooth spheres was quite different from that which occurred in a bed of rough beads.

\section{Particle Deviations from Streamlines of Flow}

As a first approximation it is reasonable to assume that resistance to flow through a porous medium ought to increase as a simple function of the amount of entrained material in the inlet stream that becomes deposited. For this reason, many researchers have studied factors affecting both deposition probabilities and the consequences of that deposition on permeability.

There are several contributing mechanisms to consider when particles are drawn by flow through a porous material. To a first approximation, particles in suspension will tend to follow the streamlines of flow that pass through a porous material. According to trajectory theory, the likelihood of particle collision with a surface within a deep bed filter can be estimated based on interception, taking into account that particle movement will be affected not only by the streamlines of liquid flow, but also by sedimentation. Also, one can expect a lubrication effect whenever the streamline followed by a specific particle passes close by a solid surface. Such a particle will have a tendency to deviate from the streamline passing through its center in order to avoid close approach to a surface (Goren and O’Neill 1970). Some researchers have used the term "lift forces" when describing the tendency of suspended particles in a laminar, flowing suspension to move away from solid surfaces (Saffman 1965). As illustrated in Fig. 3, a consequence of this mechanism is that particles undergoing laminar flow tend to migrate away from the walls of pores. For completeness, effects of gravity and momentum also need to be considered here, especially if the particles are relatively large.

Further deviations from streamlines of flow can be expected to result from random thermal motions, i.e. Brownian motion (Davis 1992; Kulkarni et al. 2005; Iliuta and Larachi 2006). The thermal motions become increasingly important with decreasing size of suspended particles, especially if the diameter is less than about $0.1 \mu \mathrm{m}$. Brownian motion of small particles provides the main mechanism by which they can diffuse in various directions even in the absence of flow. Gel permeation chromatographic methods (Helfferich and Carr 1993; Larson et al. 2003; Barrande et al. 2007; Siu et al. 2007) take advantage of both streamline-induced flow and Brownian diffusion, since the former mechanism (streamline flow) can provide the main transportation mechanism through the column, and the latter mechanism (thermal motions) provides a way for sufficiently small macromolecules or particles to linger within small capillary spaces, an effect that tends to increase their average elution time compared to larger macromolecules or particles.

\section{Flow Rate Effects}

Further insights relative to the causes of permeability reduction have been gained through studies involving different flow rates of liquids or suspensions through porous media. Various studies have reported that increases in flow resistance, for a given volume of suspension passed through media, tended to become less severe with increasing flow rate (Kau and Lawler 1995; Narayan et al. 1997; Veerpaneni and Wiesner 1997; Mays and Hunt 2005). This is possibly due to less deposition (fewer collisions or less 
sticking tendency in the presence of higher shear) and a more even spread of particles through the depth of the filter. One way to explain a lower frequency of collisions is that the higher flow rates may favor "hydrodynamic avoidance" of contact between the particles and the substrate surfaces (Brenner 1968), making it less likely that particles get stuck in positions where they obstruct flow. This tendency is illustrated in Fig. 4. According to this suggested mechanism, a particle being drawn towards a solid object by laminar flow will tend to avoid that object by moving away from streamlines that come too close to the object. As a consequence, a higher proportion of the particles stay towards the centers of flow channels, thus avoiding some of the places where they might have contributed to a greater loss of permeability through the media. By contrast, a lower flow rate would be expected to increase the relative importance of Brownian motion, which would be expected to allow particles to become stuck in a wide variety of locations, even within or at the entrances of pore openings that are transverse to the main flow. As will be discussed later, an alternative way to account for such observations is to envision hydrodynamic forces becoming increasingly effective at higher flow rates in scouring the surfaces within channels of flow, detaching particles that may tend to inhibit flow.

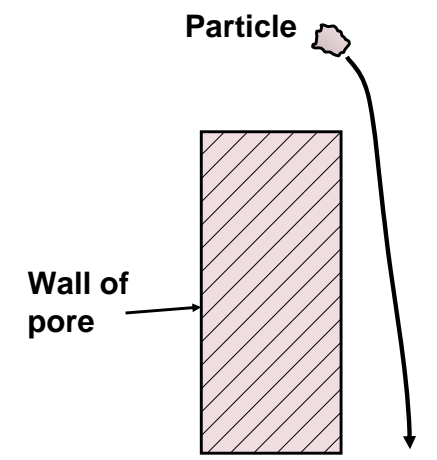

Fig. 3. Particle migration perpendicular to shear at a fixed surface, a mechanism that favors particle transport away from walls

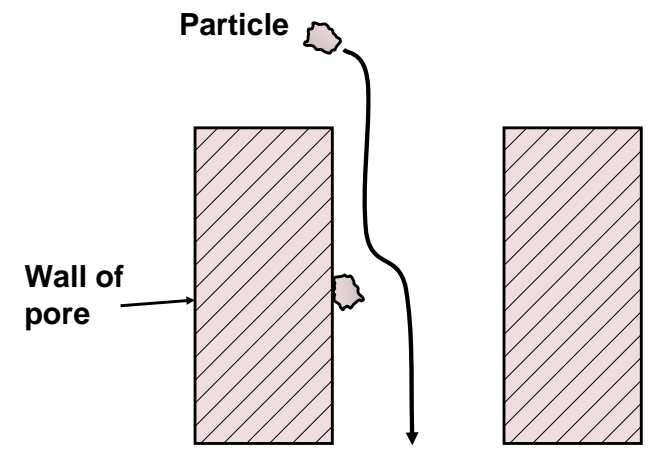

Fig. 4. Hydrodynamic avoidance, a mechanism that reduces the likelihood of collisions with objects protruding from pore walls

\section{Hydrodynamic blocking}

Some investigators have attributed decreasing rates of permeability loss at higher flow rates to hydrodynamic blocking by already-deposited particles (Putnam and Burns 1997). The idea is that at slower rates of perfusion through a porous medium, a suspended particle would have a higher probability of accumulating on the surface of other such particles that already are present on the pore walls. By contrast, at higher flow rates the lubrication effects, as described in the previous section, would be expected to be more dominant, tending to make the particles pass around obstructions.

In some instances higher rates of fouling have been observed at higher flow rates or higher applied pressures during filtration of suspensions (Iliuta and Larachi 2003; Mourouzidis-Mourouzis and Karabelas 2006). One likely explanation is that the particles considered by these researchers were sufficiently large relative to the pore sizes that they were not able to pass through constricted areas of the medium, despite effects such as the hydrodynamic avoidance mechanism described in the previous section. 


\section{Entrainment}

Another possible explanation for instances of lower permeability loss (per unit volume of suspension entering the medium) is that higher flow rates might erode away some particles that are protruding outwards into the streamlines from the sides of pores (Vigneswaran and Chang 1989; Bai and Tien 2000). Such a mechanism is suggested in Fig. 5. However, even if hydrodynamic forces are able to dislodge previously deposited particles from surfaces, it would be dangerous to assume that such a mechanism necessarily would contribute to permeability. Depending on the distributions of particle sizes and pore sizes, one might expect re-entrained particles to get stuck again and block other points of constriction, further downstream in the medium.

It is reasonable to expect that the proportion of particles that can be hydrodynamically detached from a surface within porous media ought to increase with increasing rate of flow (Gruesbeck and Collins 1982; Gabriel and Inamdar 1983; Cerda 1987b; Vigneswaran and Chang 1989; Belfort et al. 1994; Kau and Lawler 1995; Bai and Tien 2000; Iliuta and Larachi 2006). Sudden changes in flow may tend to jostle fine particles so that they can be entrained by the prevailing flow (Cleasby et al. 1963; Fitspatrick 1993). In addition, studies have shown that a given rate of flow past a surface is more likely to detach larger particles, compared to smaller particles (Ison and Ives 1969; Hubbe 1985; Cerda 1987a; Bai and Tien 1997; Burganos et al. 2001). All of these factors can be expected to affect whether suspended particles are likely to plug channels within porous media.

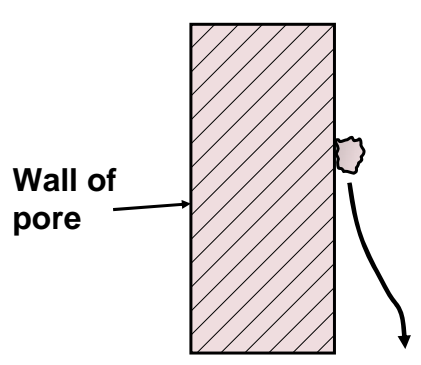

Fig. 5. Entrainment by hydrodynamic shear, an event that increases in probability with increasing shear stress and particle size

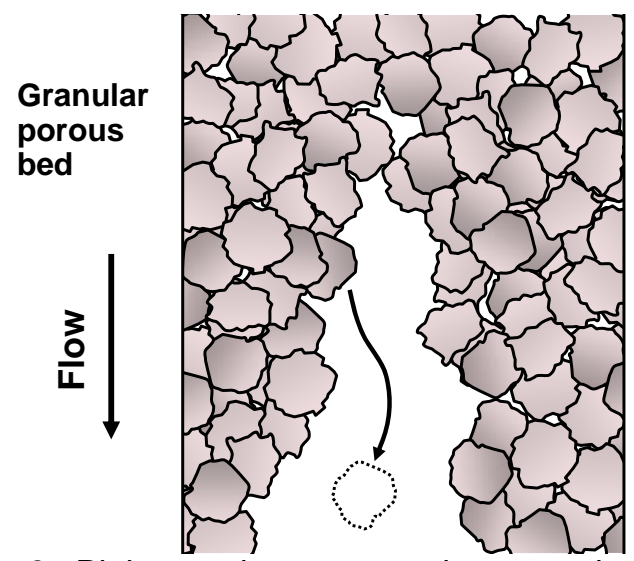

Fig. 6. Piping, a phenomenon that sometimes occurs at the down-stream side of a packed bed exposed to flow

\section{Erosion and piping}

Piping is a term used for one kind of particle entrainment that actually can increase the permeability of a bed of granular material (Ginn et al. 1992; Khilar and Fogler 1998). The mechanism is illustrated in Fig. 6, where one envisions the loss of granular media from parts of a bed of granular media. Piping can occur in some cases if channels are able to form and open out at the down-stream side of the media. Erosion of particles from the sides of such a channel tend to make it larger, as increasing amounts of the bed material transported out of the bed. Piping might be expected, for instance, if there is a hole in a screen supporting a packed bed of granular filter media. Ginn et al. 
(1992) used data to show that relatively large channels, which they called "worm holes," can exist within a backed bed of sand or coal particles.

\section{Chemistry-Related Effects}

The discussion up to this point has focused on physical and mechanical effects, but the surface chemistry and the electrically charged nature of surfaces also can play large roles relative to permeability loss of porous media. As evidence of this, researchers have found instances in which permeability loss was brought on or made more severe by changes in $\mathrm{pH}$ (Iritani et al. 1995).

In addition to the hydrodynamic forces already discussed, small particles also can become released from solid surfaces as a result of changes in aqueous solution properties. The phenomenon is sometimes called peptization (Reed 1980; Gabriel and Inamdar 1983; Khilar 1983; Khilar and Fogler 1984, 1998; Sharma et al. 1987; Sharma and Yortsos 1987a; Cerda 1987a,b; Vaidya and Fogler 1990; Ryan and Elimelech 1996; Bai and Tien 2000; Iliuta and Larachi 2006). A more descriptive term for this phenomenon is "release induced by changes in ion concentrations." The mechanism of peptization is illlustrated in Fig. 7, which depicts typical force-distance curves for interaction between two solid surfaces. The lower, solid line in Fig. 7 (both in frames A and B) represents the everpresent dispersion component of van der Waals attractive forces. The dashed line in frame A represents a component of repulsive electrostatic forces in a hypothetical initial condition, where it is assumed that the aqueous solution is saturated with salt ions, as a consequence of long-term equilibration with the surrounding mineral surfaces. The dotted line represents the change in Gibbs free energy due to interactions between the surfaces as a function of distance of separation. As shown in Fig. 7A, under such conditions it is reasonable to expect that a small particle can be trapped in a "well" of free energy of interaction. The likelihood that such a particle will diffuse away by Brownian motion from the surface can be exceedingly low, depending on the detailed description of the situation.

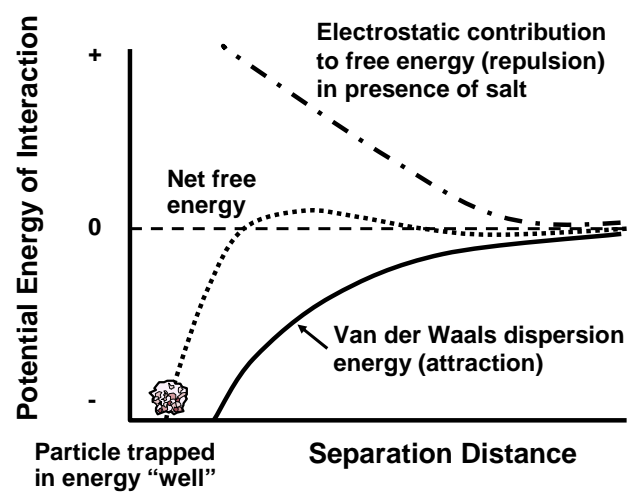

Fig. 7A. Representation of interactive free energy as a function of separation distance for a particle trapped in a "well" of interactive free energy in the presence of salt ions

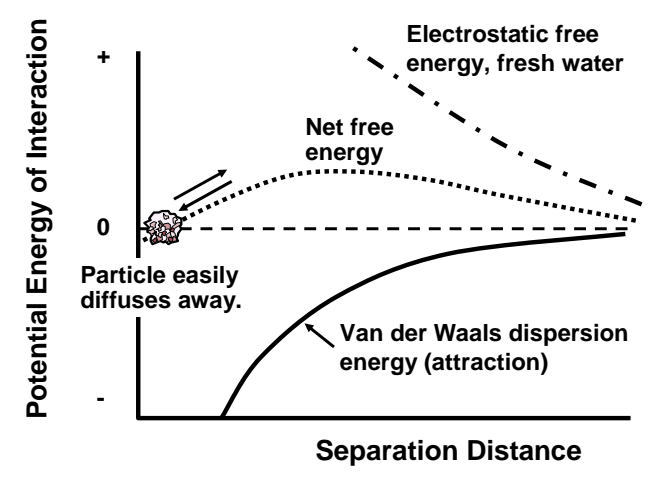

Fig. 7B. Representation of interactive free energy as a function of separation distance after replacement of the suspending solution with fresh water, making peptization likely 
Figure 7B depicts a contrasting situation, representing what might happen if the aqueous solution were replaced by salt-free water. Notice that the attractive van der Waals forces are expected to stay the same. However, the magnitude and range of the electrostatic forces can be expected to be much reduced in comparison to the lower concentration of salt ions. As a result, this hypothetical example indicates that the "well" of free energy has almost disappeared in the hypothetical case considered. Thus, it is reasonable to expect that such a particle can easily be detached or drift away from the surface to which it had been clinging, even without significant application of hydrodynamic shear.

\section{Water sensitivity}

Evidence of the peptization mechanism, as just described, has been noted many times, especially in the scientific literature related to enhanced oil recovery (Johnston and Beeson 1945; Jones 1964; Mungan 1965; Hardcastle and Mitchel 1974; Muecke 1979; Khilar 1983; Khilar et al. 1983; Khilar and Fogler 1983, 1984, 1997, 1998; Goldenberg 1985; Cerda 1987a,b; Sharma et al. 1987; Sharma and Yortsos 1987c; Vaidya and Fogler 1990; Mojarad and Settari 2007). Severe reductions in permeability have been reported most often in cases where fresh water was injected into an oil well in an effort to increase the groundwater pressure and force oil to flow toward an adjacent borehole. The effect has been almost universally attributed to the presence of fine clay particles, including the platy, easily delaminating montmorillonite clays in the formation. The permeability reductions, in such cases, have been attributed to downstream migration of clay particles into positions where they get strained by constrictions in the network of pores. Because the straining mechanism depends on physical entrapment, rather than adhesion, one can expect such problems to become more serious when conditions are changed to increase the repulsive electrostatic forces between surfaces.

\section{Agglomeration}

Rather different behavior can be expected in cases where the fine particles tend to be much smaller than the narrowest parts of the porous system. Even if the particles in a suspension are too small to clog pores by themselves, studies have shown that agglomerates formed by such particles can be quite effective in reducing the overall permeability of the medium (Tien et al. 1979; Payatakes et al. 1981; Darby and Lawler 1990; Kau and Lawler 1995; Iliuta and Larachi 2006).

The suspected mechanism is illustrated in Fig. 8. Part A of the figure represents a simple pore and the passage of a particle that is clearly smaller than the dimensions of the most restricted zone. Especially if one assumes the same sign of electrical charge on all of the surfaces, leading to electrostatic repulsion, it is reasonable to expect that the particle will pass through the pore with no effect on permeability. However, as illustrated in Fig. 8B, an agglomerate comprised of many such particles could be expected to clog the same pore. Results can be expected to depend on both the porosity of the agglomerate (Shapiro et al. 2007) and whether or not it can deform to seal itself against the pore walls in the location where it becomes trapped by hydrodynamic forces. 


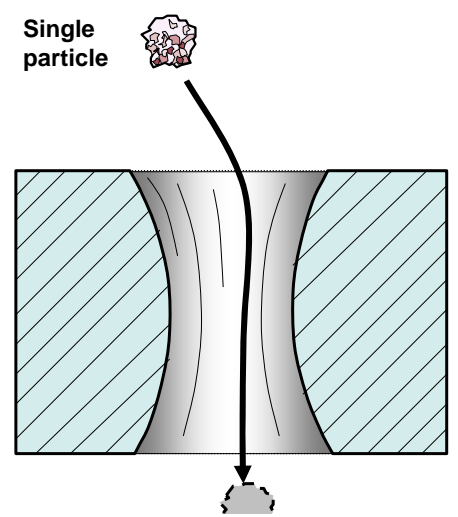

Fig. 8A. Consider a case in which individual particles are too small to be retained by straining at constricted sites.

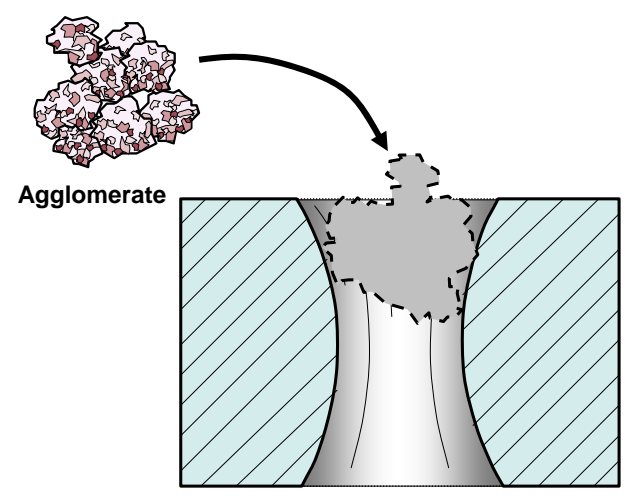

Fig. 8B. An agglomerate of small particles may become large enough to become physically stuck at a narrow point in a pore network.

Payatakes et al. (1981) proposed that a mechanism such as that illustrated in Fig. 8 may be initiated by gradual deposition of fine particles within porous media, such that agglomerated masses of fine particles may be grouped together at points within the bed. Then, as a result of changes in flow or of electrolyte conditions, agglomerates of particles can become entrained in flow. As suggested in the figure, an agglomerate of small particles may be much more likely to clog constricted areas in the bed, compared to the individual fine particles. Evidence that agglomerates of deposited particles can become suspended and pass into the effluent from a packed bed filter under conditions or ordinary water treatment was obtained by Ginn et al. (1992), who used a laser refraction particle size instrument to quantify particle size.

An important early milestone in the study of polyelectrolyte interactions was reached in a publication by Smellie and La Mer (1958). These authors discovered that complexes formed by interaction of soluble positive and negative species yielded precipitation of polyelectrolyte complexes, which tended to impede the dewatering of phosphate slimes. The study was important in laying a foundation for our present understanding of polyelectrolyte complexes and polymer bridging. Skerlos et al. (2001) considered a case in which a similar mechanism could involve complexation interactions between sparingly soluble block copolymers, resulting in entities larger than passages through a filter medium.

\section{Deposition (adhesion mechanism)}

Considerable evidence has been reported concerning an effect that is almost the opposite of that which was just discussed; cases in which permeability loss was brought about by the adhesion of small particles to the walls of pores (Ives and Pienvichitr 1965; Vidyanathan and Tien 1987; Boller and Kavanaugh 1995; Herroro et al. 1997; Kuhren et al. 2000; Howe and Clark 2002; Iliuta and Larachi 2005; Kilduff et al. 2005). In other words, the researchers were dealing with situations in which the suspended particles were believed to be much smaller than the pores. One of the ways in which such deposition can be expected to increase resistance to flow is by increasing the wetted surface area inside the filter. In addition, the average pore size is decreased as a result of such 
deposition. Figure 9 provides a simplified representation of how such adhesion might be expected to result in monolayer coverage of pore surfaces, thus decreasing the crosssectional areas available for flow. Such a pictorial model is especially appropriate for understanding permeation reduction in cases where the suspended particles are attracted to the substrate, but where they are not attracted to each other. Examples of such behavior are found especially in the filtration of bacterial cells and similar suspensions through coarse filter beds (Rijnaarts et al. 1996; Herroro et al. 1997; Iliuta and Larachi 2005, 2006).

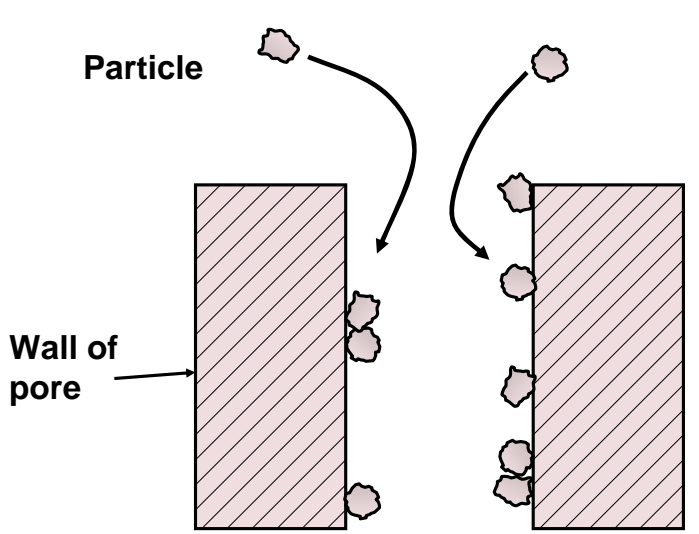

Fig. 9. Classical mechanism of monolayer deposition of small particles onto pore walls, decreasing the open area of the pore

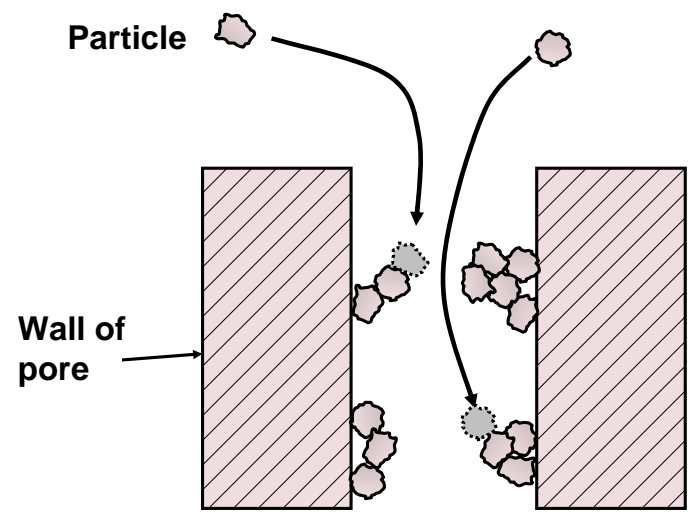

Fig. 10. Classical mechanism of multilayer deposition of small particles onto pore walls, often leading to rapid pore blockage

\section{Colloidal instability and agglomeration}

Evidence to support a mechanism such as that shown in Fig. 9 can be found by considering the interactive forces between suspended particles and pore surfaces (Herzig et al. 1970). For instance, the electrostatic forces acting between the surfaces usually are controlled by such variables as $\mathrm{pH}$, ionic strength, and the concentrations of multivalent ions (Hiemenz and Rajagopalan 1997). A variety of studies have shown cases in which permeability loss was initiated or made more severe by changes in the aqueous conditions that favored adhesion between suspended particles and the filter media surfaces (Rausch and Curry 1963; McDonough et al. 1992; Zhang et al. 2003; Singh and Song 2005, 2006; Song and Singh 2005; Gironès et al. 2006; Katsoufidou et al. 2008).

\section{Multilayer deposition}

Changed aqueous conditions also may encourage suspended particles to adhere to each other, including other particles that already have deposited within a porous medium (Khunen et al. 2000). The expected result may be multilayer deposition, as depicted in Fig. 10. An important distinction to make is that multilayers of deposited particles provide a mechanism by which particles that are much smaller than pore openings have the potential to bridge the cross-sectional area of a pore. Multilayer adsorption has been proposed in certain cases to account for increased losses in permeability with increased ionic strength of the suspending medium (Kuhnen et al. 2000; Kulkarni et al. 2005). Because conditions favoring multilayer deposition also would be expected to favor 
agglomeration of the entrained particles, it is likely that both mechanisms would occur simultaneously.

The term "ripening” has been used to describe cases in which deposition of pioneer particles within a porous medium increase the efficiency of particle collection, often increasing the rate of permeability decline as well (Darby and Lawler 1990). Such phenomena have been attributed to colloidal attraction between the particles, especially if such adhesion is much more favorable than adhesion of a particle directly to a pore surface. Darby and Lawler (1990) found that certain sizes of particles were more likely to be involved in this kind of mechanism.

\section{MATHEMATICAL DESCRIPTIONS AND FITTING}

Without mathematical analysis it can be more difficult to make practical use of concepts such as those introduced, in qualitative terms, in the previous section. Fortunately, in the field of permeability reduction there has been substantial effort to derive and to evaluate empirical and theoretical relationships (Hermans and Bredée 1936; Ives and Pienvichitr 1965; Ison and Ives 1969; Herzig et al. 1970; Sakthivadivel et al. 1972; Fan et al. 1985b; Sharma and Yortsos 1987b; Rege and Fogler 1988; Sahimi et al. 1990; Imdakm and Sahami 1991; Davis 1992; Belfort et al. 1993, 1994; Bowen et al. 1995; Khilar and Fogler 1998; Pandya et al. 1998; Le Coq and Silvy 1999; Ho and Zydney 2000; Boerlage et al. 2002; Bradford et al. 2003; De Bruijin et al. 2005; Mays and Hunt 2005; Purkait et al. 2005; Berube and Lie 2006; Duclos-Orsello et al. 2006; Bolton et al. 2005, 2006; Parry 2006; Alvarez et al. 2007; Iritani et al. 2007; Mojarad and Settari 2007). Further related work pertaining to the field of papermaking science was cited in an earlier review article (Hubbe and Heitmann 2007).

\section{Pore Wall Accumulation}

Possibly due to the intriguing nature of the topic, there has been considerable work devoted to an understanding of suspended particles that are substantially smaller than typical passageways in porous media. Because the deposition of such particles cannot be attributed to mechanical straining, the topic is perhaps an ideal challenge for industrial technologists and their counterparts at universities. In general terms, researchers have gravitated towards either of two main assumptions regarding adhesion between surfaces. On the one hand, mathematical relationships can be derived based on an assumption that suspended particles can adhere to pore walls, but that they tend to avoid each other. Such an assumption is consistent with coverage of pore surfaces with a monolayer or sub-monolayer of particles (O’Melia and Ali 1978; Chiang and Tien 1985; Vidyanathan and Tien 1987; Hampton et al. 1993; Johnson and Elimelech 1995; Bai and Tien 2000; Boerlage et al. 2002; Bolton et al. 2005). A good example of this mechanism has been described in the filtration of humic acid and sodium alginate (Karsoufidou et al. 2008). An irreversible reduction in the permeability of membranes exposed to such suspensions was attributable to monolayer coating of the pore surfaces. On the other hand, one may assume that a particle in suspension can deposit onto the surface of an already-deposited particle; such an assumption is consistent with multilayer deposition 
(Payatakes et al. 1981; Chiang and Tien 1985; Darby and Lawler 1990; Choo and Tien 1995b; Kau and Lawler 1995; Kulkarni et al. 1995; Rijnaarts et al. 1996; Narayan et al. 1997; Kuhnen et al. 2000; Bradford et al. 2004; Nascimento et al. 2006; Katsoufidou et al. 2008).

\section{Monolayer deposition in pores}

As was illustrated in Fig. 9, simple adhesion to pore walls by particles much smaller than the pore diameter can be expected to decrease the effective pore diameter in a steady, non-catastrophic manner (Chiang and Tien 1985). Some authors have used the term "standard pore blocking" to describe this mechanism (Purkait et al. 2005). In this kind of model, permeability depends on the area available for deposition of suspended particles and on the amount of suspension passing that area (Datta and Redner 1998). In the case of a cylindrical pore the relationship between permeability and the effective pore radius follows from the Hagen-Poisseuille relationship (McCabe et al. 2005),

$$
\Delta P=8 v \mu L / R^{2}
$$

where $v$ is the average velocity of the fluid, $\mu$ is the dynamic viscosity, $L$ is the wetted length, $R$ is the radius of the capillary, and $\Delta P$ is the change in pressure. Because of the squared inverse dependency of $\Delta P$ on $R$, one can expect there to be a disproportionate contribution from the most restricted sections in a network of interconnecting pores.

A general equation for permeability of porous media is given by Darcy's law (See Khilar and Fogler 1998; Mays and Hunt 2005),

$$
k=-v \mu /[\rho g(\Delta H / \Delta y)]
$$

where $v$ is the approach velocity (same cross-sectional area of flow but no media present), $\mu$ is solution's dynamic viscosity, $\rho$ is solution density, $g$ is gravitational acceleration, and $(\Delta H / \Delta y)$ is the change in hydrostatic head with distance through the filter media. Darcy’s law also can be expressed in terms of the pressure difference,

$$
\mathrm{d} V / \mathrm{d} t=k A \Delta P_{\mathrm{f}} /(\mu L)
$$

where $V$ is the filtrate volume at time $t, A$ is the cross-sectional area available for flow (disregarding the presence of a solid phase), $\Delta P_{\mathrm{f}}$ is the pressure drop across the permeable material, $\mu$ is the viscosity, and $L$ is the linear length of the column through which the fluid passed through the permeable material.

Maroudas and Eisenklam (1965b) based their analysis on the Kozeny-Carman equation (Kozeny 1927; Carman 1938, 1939), which can provide a starting point for estimating the flow resistance through a well-characterized packed bed. In this kind of analysis the effect of particle deposition can be treated mathematically as an increase in radius of the filter grains, keeping their centers at the same locations. Bolton et al. (2005) applied a similar approach to predict permeability decline in the case of a fibrous filter medium. A commonly cited form of the Kozeny-Carman equation is given in Eq. (4), 


$$
\Delta p / L=150 \frac{V_{o} \mu(1-e)^{2}}{\Phi^{2} D_{p}{ }^{2} e^{3}}
$$

where $\Delta p$ is the head loss (change in pressure), $L$ is the straight-line distance through the bed, $V_{o}$ is the fluid velocity in the absence of packing material, $\mu$ is the fluid's dynamic viscosity, $e$ is the fractional void volume, $\Phi$ is the sphericity of particles in the bed, and $D_{p}$ is the particle diameter that defines the packed bed (McCabe et al. 2005; see also Carrier 2002; Chapius and Auberton 2003).

As small particles deposit individually onto pore walls, they can be expected to increase the effective surface area within the medium. O’Melia and Ali (1978) assumed that permeability decreases as the square of surface area. The specific area for a filter subject to deposition of small particles on pore surfaces was derived,

$$
A_{s}=6(1-e)\left[\left(1 / d_{c}\right)+\beta^{\prime} N A_{p} /\left(\pi d_{c}^{3}\right)\right]
$$

where $e$ is the porosity, $d_{c}$ is the diameter of collector particles, $\beta$ ' is an empirical coefficient used for fitting the data (possibly expressing the efficiency with which particles take part in the proposed mechanism), $N$ is the number of collector particles, and $A_{p}$ is the surface area of one particle.

Johnson and Elimelech (1995) carried out a related analysis that considered the effects of electrostatic repulsive forces between the particles. The kinetics of deposition were calculated based on a random pattern of collisions, while at the same time ruling out deposition at sites already occupied, plus an additional "excluded area" near to alreadydeposited particles. Kuhnen et al. (2000) compared Langmuir adsorption and random sequential adsorption models to fit data for filtration of iron oxide with a sand bed. Though this study successfully accounted for the effect of ionic strength on the extent of excluded area, the authors did not evaluate the extent of permeability loss.

Rather than finding fault with the mechanism of monolayer buildup on pore surfaces, a number of more recent studies have incorporated such deposition into two or three step processes, involving additional mechanisms (see next sections) (Hampton et al. 1993; Kuhnen et al. 2000; Zhao et al. 2004), or alternative mechanisms, depending on such variables as the ionic strength of the supporting electrolyte (Purkait et al. 2005; Katoufidou et al. 2008).

\section{Multilayer deposition in pores}

Empirical relationships have been proposed to account for the contribution of multilayer deposition to permeability loss. Gruesbeck and Collins (1982) proposed the relationship

$$
K / K_{o}=\exp \left[-a \sigma_{2}^{4}\right]
$$

where $K / K_{o}$ is the ratio between the final permeability and the initial, unobstructed permeability, $a$ is a constant of the porous medium, and $\sigma_{2}$ is the amount of the deposited 
material (see Khilar and Fogler 1998). In order to deal with the specific case of peptization and water sensitivity, as described earlier, Khilar and Fogler proposed

$$
K / K_{o}=\left[1-\left(B \sigma_{2} / \sigma_{10}\right)\right]^{2},
$$

where $B$ is a constant, and $\sigma_{10}$ is the initial amount of particles that potentially can be released from pore surfaces from positions in which they presently do not impede flow.

A common feature of equations (6) and (7) is that they both predict acceleration in the severity of permeation reduction if the amount of deposit is increased in a steady manner. Another way to express this kind of behavior involves the concept of a clogging threshold (Datta and Redner 1998); in other words, there may be a critical amount of deposited particles that essentially obstructs all of the possible ways that fluid can pass through a porous medium, and permeability decreases very rapidly as the threshold is approached.

It becomes difficult to justify simple equations to fit permeability loss data in situations when the patterns of multilayer accumulation are complex. Payatakes et al. (1981) observed that particles accumulated in the throat areas and also as "pillars" facing into the flow. The effects of such structures, as well as the partial collapse of such structures on permeability is inherently very difficult to predict. Several groups have noted the importance of clogging related to the break-off of agglomerates of particles, which subsequently can be expected to have a high likelihood of clogging pores having smaller openings than the agglomerates (see next section).

\section{Plugging of Pores}

Plugging will be defined here as a mechanism in which one or more particles get stuck within a pore is such a way that there is contact with at least two opposite points on the pore wall. One can imagine several idealized cases. First, it might be assumed that a particle either exactly matches the cross-sectional opening of a pore, or it is sufficiently deformable under the applied pressure, so that flow becomes sealed off completely through the pore under consideration. Alternatively, and usually more realistically, one can assume that contact between the particle(s) and the pore walls (or among the clogging particles) is incomplete, so some fluid can leak through, albeit with higher resistance to flow in comparison with the unclogged pore.

\section{Complete pore plugging}

Researchers who employ relationships based on a pore plugging mechanism most often assume that particles are physically trapped when they encounter passages that are too small to permit passage (Herzig et al. 1970; Hampton et al. 1993; Datta and Redner 1998; Jiang et al. 2003; Purkait et al. 2005). Hermans and Bredée (1936) may be credited with introducing the concept of complete blocking of pores. In other words, as a first approximation, one assumes that if a particle is too large to fit through the throat of a pore, the flow through that pore goes essentially to zero. It follows that flow through the network of pores needs to be redirected and local flow velocities through still-open pathways may need to increase in order to maintain a certain overall flow through the media. 
Although exponential relationships between filtrate volumes and filter bed permeability have been proposed based on a concept of complete pore plugging (Hermans and Bredée 1936; Boerlage et al. 2002), such mathematical predictions must be viewed with caution. Consider, for the sake of discussion, a very simple porous system consisting of 100 parallel, equal, cylindrical pores, and a volume of suspension that contains a distribution of particles some of them big enough to completely seal off some one of those pores. When the first particle becomes lodged within a pore, the permeability of the system decreases by $1 \%$. Since flow no longer passes through that pore, there is no chance that a second particle will enter that pore. When the $99^{\text {th }}$ particle gets stuck in the next-to-last open pore, the remaining permeability of the system decreases by half. When the $100^{\text {th }}$ particle gets stuck in the last pore, the permeability decreases abruptly to zero. Whether or not this description of a complete-blocking process can be regarded as being realistic, the model suggests that almost complete blockage will be associated with a certain "saturation" level of filtrate having passed through the media, leaving behind those particles big enough to serve as plugs (Datta and Redner 1998). Hampton et al. (1993) predicted that results should be extremely sensitive to the detailed distributions of pore sizes and particle sizes.

\section{Partial pore plugging}

Some investigators have considered mechanisms in which particle clogging of pores does not completely stop the flow (Matsumoto et al. 1992; Choo and Tien 1995a; Shapiro et al. 2007). As noted by Shapiro et al. (2007), partial clogging of a pore within packed media exposed to suspended particles implies that further clogging of that pore is likely. Thus, if a partially clogged pore still has an open connection to a wider network of pores, then one can expect flow to transport additional particles to the clogged area. In comparison to the original pore cross-section, the clogged area can be expected to act as a sieve, trapping yet smaller particles in the remaining throat areas between the original pore walls and the surfaces of earlier-deposited particles.

It is reasonable to expect that the compactness with which particles become deposited with pores, and hence the effectiveness of the lodged particles in impeding flow, ought to be influenced by forces of repulsion or attraction between the surfaces. Support for such a mechanism, at least when it happens at a larger scale, can be found in studies of the volume of sediments in relation to the mass of solid materials (Philipse and Wierenga 1998; Faibish et al. 1998; Wang and Siu 2006; Dunleavey-Routh and Vincent 2007). An underlying assumption for such a mechanism is that deposited particles do not move to a significant extent from their initial points of attachment, probably due to the development of very strong inter-surface forces, including van der Waals forces. In the presence of electrostatic repulsion forces, which often have an effective range that is greater than the van der Waals forces, the surfaces are able to slide past each other during the process of deposition to achieve a higher density of packing.

Wiesner (1999) proposed that particle deposition processes could be grouped into two limiting classes, either "ballistic" or "random." The ballistic processes were predicted to form denser, less permeable sediment layers, whereas random sticking collisions can be expected to result in a more open, permeable structure of deposited particles. Such considerations can be expected to apply not only with respect to clogging 
within the pores of a filter medium, but also during the formation of a cake at the surface of a filter (see next section).

\section{Cake Buildup (on a membrane, for instance)}

Cake filtration can be defined as a process in which the material that is being filtered gradually builds up at the entrance to a filter medium (Hermans and Bredée 1936; Heertjes 1957; Mays and Hunt 2005; Parry 2006). Cake filtration, including effects of a "surface film" can be especially important in various applications of membrane filters (Matsumoto et al. 1992; McDonough et al. 1992; Murase et al. 1995; Carroll 2001; Howe and Clark 2002; Jiang et al. 2003; Tabara et al. 2004; Zhao et al. 2004; De Bruijin et al. 2005; Meng et al. 2005; Purkait et al. 2005; Duclos-Orsello et al. 2006; MourouzidisMourouzis and Karabelas 2006; Iritani et al. 2007; Katsoufidou et al. 2008). Tyner et al. (2006) found evidence that cake filtration can play a major role in the sealing of waste lagoons. Likewise, Bolton et al. (2005) found evidence of cake filtration even in the case of deep bed filration. The cake filtration mechanism makes sense when at least some particles within the suspension are clearly large enough to be held out from the entry pores into the original filter medium. Often the permeability of the cake can be lower, and its capture efficiency may be higher than that of the uncovered filter at the start of the process.

Figure 11 offers a pictorial representation of a cake-forming process. As the figure suggests, it is possible for cakes to form even in cases where particles in the suspension may be smaller than the pore openings in the face of a membrane. In principle, a layer of deposited particles can start to build from the edges of a pore, and the cake can be completed when "capstone" particles complete a primitive arch over the pore. Of course, cake filtration becomes much more likely when at least some of the particles or fibers in the suspension are big enough to be excluded from individual pores. Also, to be more realistic, one should consider the likelihood of a mixed mechanism in which some particles deposit onto pore surfaces, and the cake starts to build up from such particles that are attached near to the entrances of pores.

Mays and Hunt (2005) examined the results from several experimental studies, and they concluded that a cake filtration model best explained some of the data. In particular, the dependency of permeability on flow rate was consistent with the buildup of a cake on the filter surface. Other studies have shown that the rate of cake buildup can depend on the way the incoming suspension is introduced to the membrane. The term "cross-flow" is commonly used to denote cases in which the predominant flow of incoming fluid is parallel to a membrane surface, a design that sometimes can result in removal of deposited particles by hydrodynamic forces (Davis 1992; Belfort et al. 1994; Murase et al. 1995; Tarabara et al. 2004; De Bruijn et al. 2005; Berube and Lei 2006; Bessiere et al. 2008; Santos et al. 2008). By contrast, the term "dead end" is sometimes used to denote filtration operations in which the inlet flow proceeds directly into the filter medium (Davis 1992; Belfort et al. 1994; Iritani et al. 1995; Carroll 2001; MourouzidisMourouzis and Karabelas 2006; Bessiere et al. 2008; Santos et al. 2008), thus providing no mechanism to limit the buildup of a cake. 


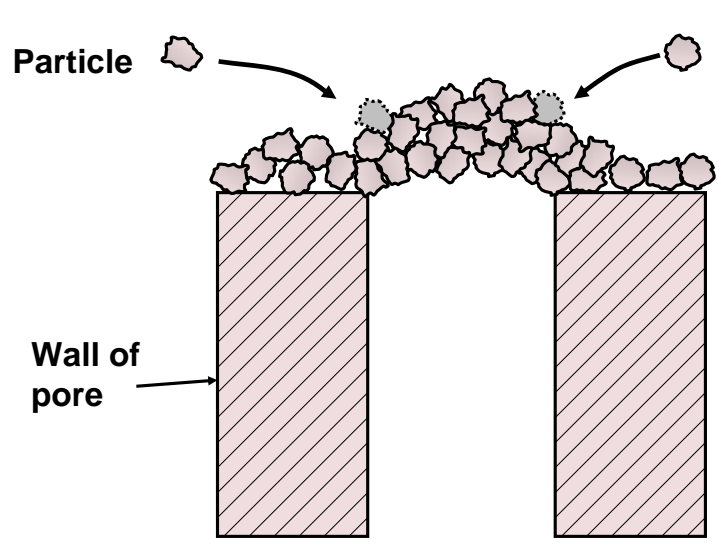

Fig. 11. Classical mechanism of monolayer deposition of small particles onto pore walls, decreasing the open area of the pore

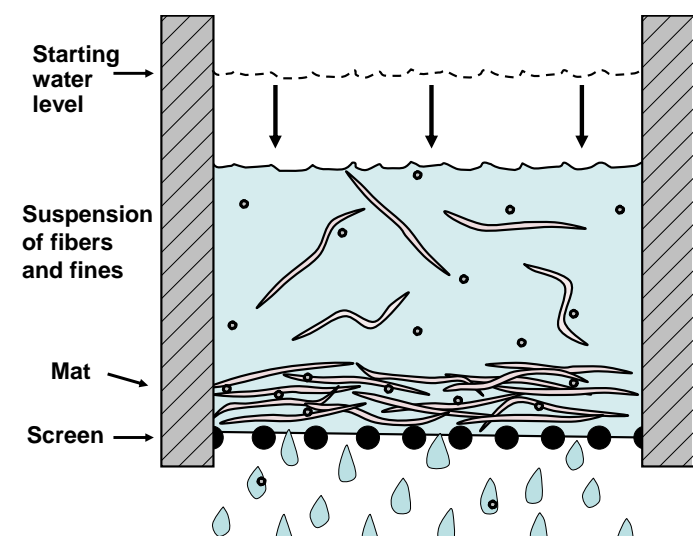

Fig. 12. Hybrid filtration mechanism during formation of paper, in which fibers in the suspension form a mat that becomes the main filter medium during the process

\section{Cake plugging}

There are several mechanisms, in addition to the build-up of more material, that can tend to make filter cakes less permeable over time. One such mechanism is pore plugging by particles that are too small to be effectively held out at the cake surface, but large enough to become filtered at constrictions within the case itself.

Other factors to consider are the compressibility of the cake (Murase et al. 1995; Mourouzidis-Mourouzis and Karabelas 2006), as well as a possibility that a cake densifies with the passage of time during continued application of pressure (Parry 2006). Singh and Song (2006) found a good correlation between cake compressibility and the fouling tendency of colloidal matter.

\section{Papermaking}

The process of forming a sheet of paper bears many similarities to the cake filtration operations just described. As suggested by the illustration in Fig. 12, there are some distinguishing features of papermaking, in comparison to ordinary cake filtration. The suspended matter used in papermaking usually contains a mixture of fibers and much smaller solids, i.e. "fines." The fibers are long enough that almost all of them are retained when the sheet is dewatered on a forming fabric. By contrast, the fines can be retained only by the mat of fibers itself, as it is being formed. Either the fines can be mechanically sieved by the fibers, or the fines may be bound to the fibers through the action of colloidal forces, including the possible action of polymeric retention aids and other chemical additives.

As was noted earlier, the mechanism suggested in Fig. 12 describes only part of what happens during the formation of a paper sheet; a full description would need to consider effects of pulsations of pressure and flow, for instance. An earlier review article described how various papermaking operations, furnish characteristics, and related chemical treatments can affect the relative permeability of wet paper as water is being removed from it (Hubbe and Heitmann 2007). There is evidence that such pulsations can cause the release of fine particles (Zeilinger and Klein 1995); thus, this "washing" action can be part of the explanation for an enhancement in dewatering rates by releasing such 
fines from positions where they impede flow through pores within the wet web of paper (Räisänen 1996).

\section{Exponents as an Indication of Mechanism}

Boerlage et al. (2002) used the following expression to represent the main implications of several different mechanisms, as discussed in previous subsections,

$$
\mathrm{d}^{2} t / \mathrm{d} V^{2}=k(\mathrm{~d} t / \mathrm{d} V)^{\mathrm{n}}
$$

where $t$ is the filtration time, $V$ is the filtered volume, $k$ is a constant representing resistance to flow, and the exponent $n$ can be related to different idealized mechanisms that have been used to account for permeability loss. Values of $n$ equal to $0,1,1.5$, and 2 represent cake filtration, intermediate filtration, standard blocking, and complete blocking, respectively (Hermans and Bredée 1936; Kim et al. 1993; Bowen et al. 1995; Madaeni 1997; Boerlage et al. 2002). Iritani et al. (1995) found that the exponent governing resistance to flow gradually changed with the passage of time, suggesting that the mechanism may have been shifting from one dominant form to another during the course of testing. Ho and Zydney (2000) developed a unified model that was able to encompass a transition from predominantly membrane pore blockage to predominantly cake filtration.

\section{Head Loss Development as an Indicator of Mechanism}

In an effort to further account for losses in permeability, Bedrikovetsky et al. (2001) measured the pressure at different levels within deep-bed filtering media during a filtering operation. Such a procedure made it possible to distinguish the effects of the amount of deposition in a certain layer of filter media and the effectiveness of the deposited material in decreasing the permeability of that layer.

\section{Deposit Location Distribution as an Indicator of Mechanism}

Various mechanisms of particle deposition all tend to suggest that the greatest proportion of particle deposition, as well as the greatest incremental losses in permeability will be associated with the first layers of pores that the entering fluid encounters (Sharma and Yortsos 1987b; Mays and Hunt 2005; Kim and Whittle 2006; Nascimento et al. 2006). For example, Datta and Redner (1998) calculated probability distributions for the locations of deposited particles based on an assumed clogging mechanism. Because a particle will become trapped at the very first location where it cannot fit through a pore, the chance that particles become stuck within a filter bed becomes lower in an exponential fashion as one follows the predominant direction of flow.

Papermaking tends to be an exception to the rule stated in the previous paragraph. Because papermaking involves filtration of fine matter before the mat of fibers has been completely formed, laboratory and pilot-scale tests often show the highest proportion of fines present in layers nearest to the forming fabric (Tanaka et al. 1982). Such layers represent the part of the paper web that forms first and remains the most dense and least permeable during much of the sheet-forming process, especially when paper is formed by simple filtration under laboratory conditions. Consequently, it is reasonable to expect 
such layers to be effective in sieving fine matter during one-directional dewatering of a paper sheet. One should note, however, that under industrial papermaking conditions the distributions of fines in paper can be the reverse of what has just been described (Zeilinger and Klein 1995). The explanation is that modern paper machines employ hydrofoils on the opposite side of the forming fabric from the wet web of paper. The hydrofoils create so much hydrodynamic shear in the layers of fibers nearest to the forming fabric that many of the fine particles are washed out of the paper web. This situation is somewhat analogous to the effects of hydrodynamic flow causing some particle detachment during bed filtration, and thus affecting the resulting distribution of deposited particles (Bradford et al. 2007).

\section{SIMULATION OF PORE OCCLUSION AND FLUX REDUCTION}

By means of computer simulation methods it is possible to take some of the approaches considered in the previous section and apply them to mathematical representations of different assumed models to represent a network of pores (Fatt 1956; Tien et al. 1979; Todd et al. 1984; Fan et al. 1985a,b; Imdakm and Sahini 1987, 1991; Mackie et al. 1987; Rege and Fogler 1987; Sharma and Yortsos 1987b; Sahimi et al. 1990; Hampton et al. 1993; Choo and Tien 1995b; Putnam and Burns 1997; Ramachandran et al. 1998; Lee and Koplik 2001; Seminario et al. 2002; Kim and Whittle 2006; Bauer et al. 2008; Bessiere et al. 2008; Kampel et al. 2008). The idea is to track the motions and fates of numerous hypothetical particles as they progress through a mathematical representation of the porous media under consideration, obeying a preselected set of stochastic rules and probabilities. As noted by Ramachandran et al. (1998), many of the works cited in the previous section were based on continuum models. Bed properties were treated as if they were the same for flow in all directions, i.e. isotropic. The challenge is to build a bridge bewteen the microscopic mechanisms and the observable macroscopic permeability losses (Datta and Redner 1998). In particular, continuum models do not deal very well with issues related to how a porous network responds to localized clogging at different points. The permeability of the medium as a whole is highly dependent on how well the pores are connected together. A statistical approach, often called percolation theory (Sahimi et al. 1990), is used to predict both the manner in which a network may become partially clogged, and also the effect of such clogging on the overall permeability.

\section{Classifying Real Pore Networks}

The first step in putting together a mathematical representation of a porous system can entail drawing a picture of it and considering what aspects of the picture represent distinguishing characteristics. The terms granular, chromatographic, membrane, and papermaking will be used in subsequent discussion to differentiate the four contrasting types of filtering media that have been discussed in this article.

\section{Granular}

Granular filter media is used in operations called “depth filtration,” or "deep bed filtration." One of the characteristics of such operations is that there is a feedback 
mechanism between particle collection and subsequent performance of the filter (Datta and Redner 1998). In addition, clogging usually occurs mostly at the upstream end of the filter bed. Figure 13 provides a pictorial representation of a granular bed filter, through which a probe particle is passing.

Bryant et al. (1993) proposed that various effects of filtration in granular media ought to be represented by a network comprised of pore bodies and throats. For instance, the throats may be represented by symmetrically restricted zones, as was shown in Figs. 1 and 8 . To represent such a system mathematically, each enlarged opening (pore body) within the bed should be connected by a number of open paths to the adjacent pore bodies.

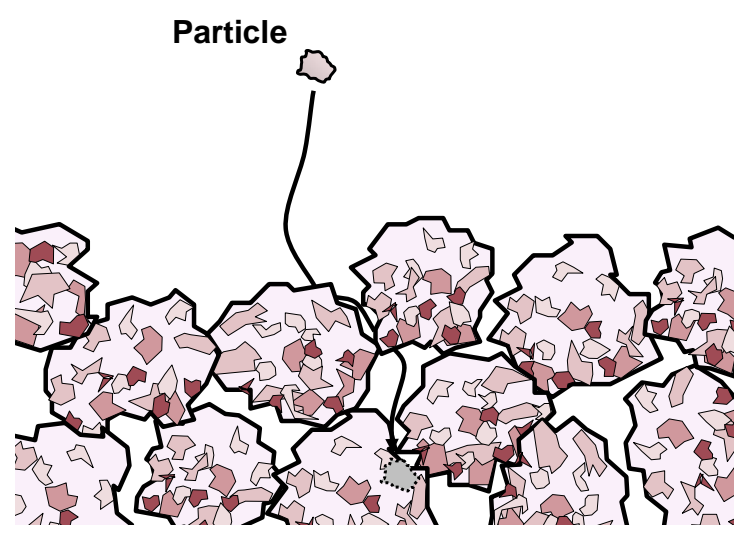

Fig. 13. Representation of a granular packed bed filter medium in which particle retention can be achieved either by mechanical straining or by interactive forces

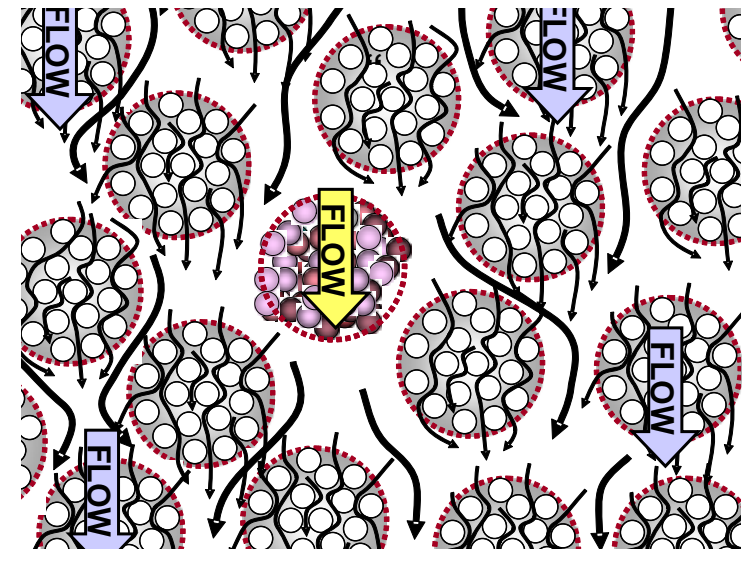

Fig. 14. Representation of a chromatographic medium in which the particles making up the bed contain networks of much smaller pores

\section{Chromatographic}

Chromatographic columns, such as those used for gel permeation analysis of the size distributions of soluble polymers, consist of beds of nanoporous particles (Linden et al. 1999). In a superficial way, a packed bed of nanoporous chromatographic packing material can seem similar to a granular packed bed, as discussed in the previous section. However, as illustrated in Fig. 14, one has to account for the fact that flow can occur not only around the component particles, but also through them, by means of networks of much smaller pores. In chromatographic applications it is often adequate to assume that most of the permeation taking place within the nanoporous particles is a result of molecular diffusion; however the presence of a pressure gradient suggests that at least part of the net flow through the system is due to liquid movement through the smaller pores. Linden et al. (1999) found evidence that individual protein macromolecules adsorbed on pore surfaces or entrances can inhibit passage of other macromolecules in such systems.

A particular challenge in dealing with flow through columns packed with silica gel and similar materials lies in differentiating between effects due to flow around vs. through the nanoporous particles. Aydin et al. (2008) addressed such problems from an experimental standpoint by completely filling in the small pores with a cross-linked 
organic polymer. In other cases, investigators have struggled to find suitable non-porous packing materials that have similar outer surface characteristics as the nanoporous packing material under consideration, in order to carry out control experiments (see, for instance, Hubbe et al. 2007).

\section{Membrane}

Though not all membranes are alike, they generally can be described by the word "thin". In addition, many membranes are manufactured to have a specified range of pore size, depending on the intended application. Figure 15 presents a pictorial representation for an especially simple membrane filter, in which the pores can be regarded as cylinders extending from one side to the other of the membrane. Such media are subject to three kinds of fouling: adhesion to pore walls, blockage of pores, and caking on top of the membrane. In terms of mathematical modeling it is reasonable to envision that reductions in the permeability of a membrane filter can occur as a series of parallel events, in which the blocking of one pore may have little or no effect on flow through a nearby pore. However, each such pore may be subject to stochastic occurrences governing the degree to which the local permeability is reduced by particle deposition.

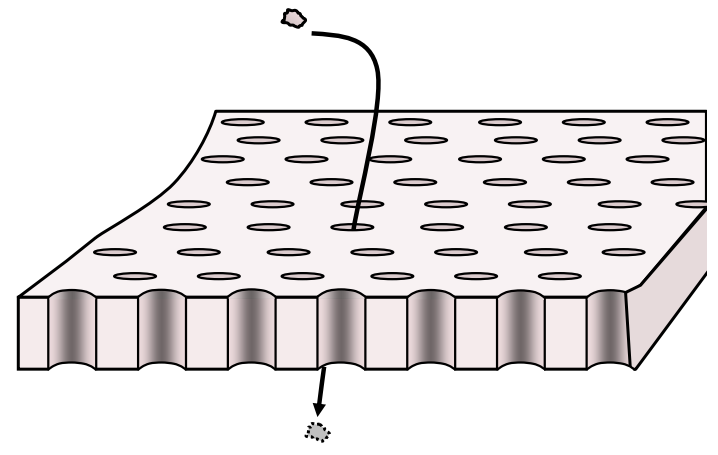

Fig. 15. Representation of a membrane filter, considering a case in which simple pores extend from one side to the other.

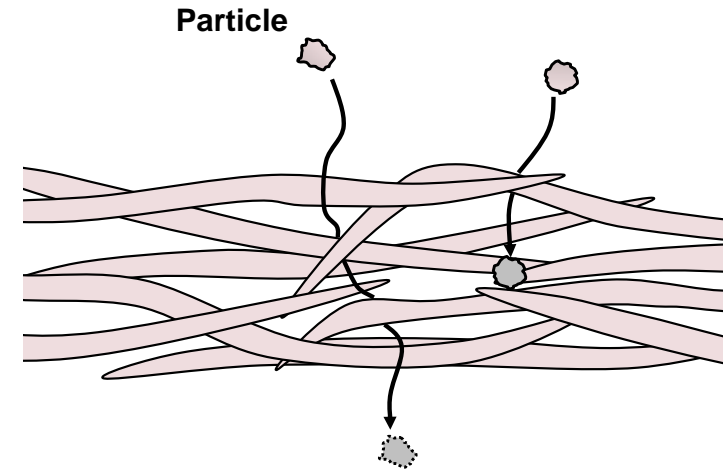

Fig. 16. Representation of a fibrous filter medium. Such media often have higher void fraction in comparison to granular packed beds.

\section{Fibrous}

Fibrous filters, including filter paper, textiles, and various screen filters, tend to have a greater proportion of void volume in comparison to granular media (Jackson and James 1986). Some of the earliest theoretical work related to permeability of fiber mats was that of Carman (1938). Concepts related to the permeability of fiber mats were further developed by researchers at the Institute of Paper Chemistry, who considered such factors as the compressibility of mats in response to applied pressure (Ingmanson 1952, 1953; Whitney et al. 1955; Meyer 1962; Han 1969). Binotto and Nichols (1979) and Zhu et al. (1995) considered related issues more recently.

Although the shapes of the filter elements are quite different from those of a granular packed bed, from the standpoint of computer modeling it still can be useful to consider fibrous media in terms of interconnected pore bodies and constrictions. For 
instance, various studies have attributed permeability reduction to a blockage of pores by cellulosic fines during the production of paper (Britt et al. 1986; Kumar and Ramarao 1996; Räisänen 1996; Hubbe 2002; Paradis et al. 2002; Cole et al. 2008; Hubbe et al. 2008). Various authors have considered network models for simulation of filtration effects vs. permeability of fibrous media (Higdon and Ford 1996; Le Coq and Silvy 1999; Clague et al. 2000).

Modeling of a paper forming process would be just the same as discussed in the previous paragraphs, except that the fiber mat is forming and densifying during the same time that it serves as a filter medium. There have been various efforts to model such events (Kumar et al. 1996; Zhu et al. 1995; see Hubbe and Heitmann 2007). It is reasonable to assume that both the pad density and the permeability of different component layers in a sheet of paper, during its formation, change continually during the process. In an effort to achieve realistic estimates of flow resistance through beds of cellulosic fibers, Zhu et al. (1995) employed micro-mechanical approach. By building upon work associated with textile technology, they were able to account for the effects of both fiber bending, fiber slippage, and changes in the distribution of fiber orientation in response to the cumulative pressure exerted on layers within the mat, as a consequence of the hydrostatic head and the flow of liquid through the mat. This approach made it possible to get a more realistic estimate of both the porosity and the permeability within different layers. The authors were able to account of moderate losses in mat permeability as a function of increasing hydraulic pressure. Though the article did not explicitly deal with effects due to unattached cellulosic fines in the flowing liquid, the mathematical approach might be adapted to serve as a starting point for such investigations in the future.

Singh et al. (2009) recently evaluated the effects of different types and levels of mineral fillers that are incorporated into paper during its manufacture. Relatively low levels of precipitated calcium carbonate (PCC), consisting either of rosettes or clustered prismatic particle shapes, tended to increase the permeability of the resulting fiber mats, presumably due to the attractive electrostatic forces between the positive PCC surfaces and the negatively charged fibers. It is reasonable to expect that interposing bulky mineral particles between fibers ought to help space the fibers apart from each other, enlarging the effective sizes of pore spaces between the fibers. Higher levels of PCC tended to reduce permeability, consistent with a clogging mechanism. Clay filler particles reduced permeability at all levels of addition; clays have a platy shape, which tends to be efficient for the blocking of pores. At the same time, except for the unexpected case of edge-wise attachment to fiber surfaces, flat mineral particles are not expected to be efficient for increasing the spaces between fibers in a wet paper sheet before it is dried.

\section{Idealized Models of Pore Networks}

Most simulation studies of filtration and permeability of porous media are based on models in which nodes are connected by various numbers of links (Fatt 1956; Rege and Fogler 1987; Sharma and Yortsos 1987b; Sahimi et al. 1990; Lee and Koplink 2001; Kampel et al. 2008). The arrays may either be ordered or random. 


\section{Capillaries}

Among the simplest ways to construct a network model for a porous system is to assume that the nodes are much larger than the dimensions of any of the suspended particles, but that they are connected together by cylindrical capillaries of uniform crosssection (Hampton et al. 1993; Choo and Tien 1995b; Kim and Whittle 2006; Santos et al. 2008).

\section{Pore bodies and constrictions (throats)}

At the next level of sophistication, the cylindrical connections are replaced by smoothly curved throats of specified dimensions (Payatakes et al. 1973; Chiang and Tien 1985; Burganos et al. 1992). Such details can be important when attempting to apply various trajectory analyses, in an effort to estimate the likelihood of particle deposition in different locations, so that the overall impact on permeability can be estimated.

Imdakm and Sahami (1991) and Burganos et al. (1992) employed trajectory analysis of the paths of many individual particles passing through simulated porous media, using a constricted pore throat model of the type just described. Hydrodynamic streamlines were considered, and the particles were allowed to enter pores in random positions. Forces and torques of various types acting on the particle were considered, and the simulation results could be fitted accurately to experimental data. This type of analysis took advantage of related work involving trajectory analysis of particle transport through or deposition within individual pores (Vidyanathan and Tien 1987). The articles by Vidyanathan and Tien (1987) and Imdakm and Sahami (1991) showed that useful predictions could be achieved by estimating the hydrodynamic torque needed to overcome the adhesive forces causing deposited particles to resist rolling from their sites of attachment.

\section{Statistics regarding plugging patterns}

For an ideal, simple membrane filter, such as that which is illustrated in Fig. 15, one can estimate the number of large suspended particles needed to plug every available channel. But the computation is more difficult in a complex porous medium. Kampel et al. (2008) considered a two-dimensional network representation. Computer simulations were carried out to determine rules to predict the maximum number of clogging events that would be needed to completely seal off flow through the bed.

\section{MINIMIZATION OF PERMEABILITY LOSS}

In comparison to the amount of research devoted to prediction and understanding of permeability loss phenomena, there has been less attention to the science dealing with ways to decrease the severity of such phenomena. Research progress related to minimization of permeability loss is discussed in this section.

\section{Removal of Fines from Inlet Flow}

As noted by Kruger (1986) and Bedrikovetsky et al. (2001), one of the most effective approaches to minimize permeability loss involves removal of particulate 
materials from incoming streams. Economic and practical considerations may or may not favor such strategies, depending on the circumstances one is dealing with. The cost to remove particulate matter needs to be low enough relative to the expected benefit for such a strategy to be worth practicing. Sometimes there can be an optimum degree of water treatment that can yield the best overall return on investment. In other cases, the main purpose of the filtration process is to remove particulate matter, so it would be like an admission of defeat to require that particles be removed in advance of such a filtration step. Nevertheless, sometimes a pre-filtering operation could be justified if such a step is able to remove components from a suspension that lead to early fouling of the main filter system (Gironès et al. 2006). For instance, simulation work by Rege and Fogler (1987) has suggested that it can make sense to remove relatively large particulate matter in such cases, especially if it is the large particulate fraction that ends up clogging the channels in a filter medium.

\section{Fines Attachment to Avoid Migration}

Papermaking applications

During the industrial formation of a paper web, one of the factors that can limit the rate of production is the rate of water removal. The presence of high levels of cellulosic fine matter in the fibrous suspension can greatly increase the resistance to dewatering, making it more difficult to operate the papermaking equipment at a profit. Though various contributing explanations have been offered for the effects of cellulosic fines on the dewatering of a paper web (see Hubbe and Heitmann 2007), it appears likely that a clogging mechanism is dominant in many case. In other words, the movements of cellulosic fines relative to the fibers in a wet mat are likely to result in the plugging of drainage channels.

One way too address problems of slow dewatering, as just described, is to bind fine particulate matter to larger particles or fibers in order to minimize migration within the filter media. Thus, during the manufacture of paper the fiber suspension can be treated with cationic polymers, binding the fines to fibers (Britt et al. 1986; Hubbe 2002; Hubbe et al. 2008). Then, once the suspension reaches the point of sheet formation, a greater proportion of the fine particles remain associated with the fibers, and less time, application of vacuum, or application of pressure is needed to coax water out of the wet paper. Not only can the rate of production be increased, but also there can be a savings in the energy that must be expended to evaporate the water that remains after the wet web has been pressed. The types of chemical additives that are particularly effective for promoting dewatering on paper machines include soluble aluminum compounds, highcharge cationic polymers, and combinations of cationic flocculants added sequentially to fiber furnish with negatively charged colloidal silica or sodium montmorillonite (bentonite) particles (see Hubbe and Heitmann 2007).

Charged additives should not be used at excessive levels, since, as shown in the early work of Smellie and La Mer (1958), an overdose of polyelectrolyte can be expected to stabilize small particles in suspension, increasing the likelihood that the filter medium becomes clogged. Related effects were reported recently by Liimatainen et al. (2009), who observed strong reductions in dewatering rates when cellulose suspensions were treated with carboxymethylcellulose (CMC). The effects were attributed to a dispersing 
effect, causing fines to remain unattached to fiber surfaces and thus more susceptible to being drawn into positions where they choke off drainage channels in the wet web of paper.

\section{Inhibitory chemicals}

Researchers dealing with enhanced oil recovery have noted that addition of multivalent cations to the injected water has the potential to suppress the release of fine particles from their points of attachment within sandstone formations. As a consequence it is possible to avoid marked decreases in permeability that would have accompanied the addition of low-salinity water without the multivalent cations. Jones (1964) found that relatively small amounts of calcium or magnesium ions were quite effective to avoid mobilization of clay particles within sandstone. Kruger (1986) listed a variety of additives that can be used in this manner, including hydroxyaluminum compounds, $\mathrm{KOH}$ (which possibly causes dissolution of aluminum from the media), and organic polymers. Some of these treatments appear to immunize sandstone beds in such a way that untreated fresh water can be used, at least for a while, without immediate plugging of the formation. The apparent mechanism involves adsorption of multivalent cations onto the mineral surfaces, thus neutralizing the electrostatic repulsion forces between the surfaces.

\section{Avoiding Unsuitable Particle Sizes}

It is reasonable to expect that a certain intermediate size range of particles should be especially harmful to the permeability of a porous medium. Particles that are many times larger than the pore spaces within the medium can be expected to simply build up as a highly permeable cake at the inlet side of the filter. Particles much smaller than the most constricted zones within the medium might be expected to pass through the filter with little effect on permeability. The latter expectation is particularly reasonable if the small particles have the same sign of charge as the porous media surfaces. Darby and Lawler (1990) found that particle size affected the probability of latex particle collection within a packed bed, but head loss was strongly correlated with the surface area of captured particles, a factor suggesting that a given mass of smaller entrapped particles will have a greater negative effect on permeability.

Based on factors discussed in this article, it is proposed that removal of either the larger or the smaller particles in a suspension with a wide particle size distribution can be desirable - removal of the large particles to minimize mechanical clogging, and removal of small particles, which, if the do become deposited within porous media, can be expected eventually to seal up the pores more effectively. If a porous media is subjected to a diverse range of suspended particles, one can expect some of the larger particles to be ideal for constricting the throat areas within porous media. Smaller particle then can be expected to become entrapped in yet more restricted passages adjacent to the first lodged particle, with the process continuing until colloidal-size particles work themselves into the smallest remaining fissures.

Particle entrapment issues can be important in the design of suitable carriers for cancer-treating medicines. An analysis by Bauer et al. (2008) showed that it can be a mistake to employ relatively large particles to transport anti-cancer drugs to tumors, despite the fact that relatively large particles can be selectively admitted to certain 
cancerous tissues, to the exclusion of typical healthy tissue. Unfortunately, the analysis showed that the large particles are prone to getting stuck too early, before they have reached the target organ.

\section{Sharp, Brief, Backflow (back-pulsing)}

Though the resuspension of fine particles within a packed bed or mineral formation usually is considered undesirable, in terms of permeability, Gruesbeck and Collins (1982) suggested a strategy to break that paradigm. They considered cases in which fine particles had become mechanically strained from a suspension, ending up by blocking constricted points in a network of pores. Their prescription was to exert a sharp, but very brief counter-flow, forcing the obstructing particles back out of their blocking positions. To minimize reblocking of the freed-up pores, the idea is to keep subsequent flow rates suitably slow in the forward direction. Amburgey and Amirtharajah (2005) and Amburgey (2005) evaluated related strategies with sufficiently gentle backflushing to avoid refluidization of filter beds; by this approach it was possible to maintain a high collection efficiency during the critical phase of filtration right after backflushing.

In membrane applications backflushing operations can be carried out with great expectations of improvements in permeability, though usually not back to the original levels (Jiang et al. 2003; Iliuta and Larachi 2005; Gironès et al. 2006; MourouzidisMourouzis and Karabelas 2006; Katsoufidou et al. 2008). Such results can be tentatively attributed to removal of much of the cake material (surface deposition), whereas much of the material deposited onto the walls of pores within a membrane filter are likely to be resistant to removal during backwashing operations.

\section{Other Strategies to Maintain Permeability}

Agitation to minimize cake buildup

The use of a cross-flow mode of membrane filtration already has been mentioned as a strategy to minimize the buildup of cake layers, which often constitute a major portion of the overall resistance to flow. But there are other related strategies. Yeo et al. (2006) described how bubbling of air can be used to agitate bundles of hollow fiber membrane media in order to maintain flux rates. It was recommended to agitate the fibers uniformly within a bundle in order to ensure an even rate of fouling on the membrane surfaces. It is likely that flow-induced rubbing between the adjacent membrane surfaces helps to dislodge cake material.

Datta and Redner (1998) suggested use of depth filter systems having a gradient of local permeability. The idea is to reduce the previously mentioned problem associated with high amounts of deposition focused in the top layers of such filters. For instance, placement of coarse media in a top layer could be used to remove part of the suspended matter, with less concern about overall permeability reduction, affecting the whole bed. Dual-media filtration systems, incorporating this type of approach, are often used for fresh water treatment. A concern, however, is that back-flushing can cause released fines to become fluidized with the finer media particles near the entrance of the bed. This process can result in further of loss of permeability if those released fines are not effectively removed from the system during the backwashing process. 


\section{INTENTIONAL CLOGGING}

There are many situations in which high permeability is considered to be undesirable. Examples include the media used to seal impoundments of liquid-containing wastes. Some strategies for dealing with such cases already have been discussed in this article. Surprisingly few researchers appear to have published scholarly work dealing with the science and optimization of such approaches.

\section{Solids of Suitable Size, Shape, and Affinity}

As noted by Rausch and Curry (1993), clay particles can be well suited for the sealing of impoundments. Results of such measures were found to depend on the colloidal stability of the clay suspensions. The present literature review did not reveal any publication in which a systematic attempt was made to identify particle attributes relative to their propensity for slowing the flow through permeable material.

\section{Polyelectrolyte Complexation}

The performance of certain filter media can suffer if the relative sizes of different channels are nonuniform. A large proportional of the flow may then pass through relatively large channels, which offer relatively poor filtration. Adjacent regions of the bed having finer pore structure may be exposed to relatively little flow. As noted by Rege and Fogler (1987), additives termed "diverting agents" have been used to selectively impede flow through relatively large channels.

De et al. (1997) pursued an innovative approach, employing in-situ polyelectrolyte complexation as a means of selectively reducing flow through relatively large channels in fiber mats. Due to hydrodynamic and macromolecular factors, high-mass cationic copolymers of acrylamide, in solution, were effectively excluded from the finer pores within such mats. The added polyelectrolytes, being restricted to the larger channels, were then able to form complexes with anionic dissolved anionic polymers and colloidal matter. The complexes thus formed tended to choke restricted zones in the larger channels of paper pulp mats, yielding an overall improvement in the efficiency of washing operations.

\section{Gelling liquids}

Related technology has been described by Durmusoglu and Corapcioglu (2000). They demonstrated the effectiveness of gelling liquids in reducing the permeability of barrier layers in impoundments. Unstable suspensions of colloidal silica were injected into filter media, such that monolithic silica gel was formed within a desired layer within the media. The relative impermeability of such layers is consistent with the principles implicit in the Kozeny-Carman analysis (Kozeny 1927; Carman 1937; Herzig et al. 1970), i.e. permeability reduction is favored by very high surface area and very small pore dimensions. 


\section{CLOSING COMMENTS}

As mentioned in the introduction, a motivation for the present review article has been to highlight concepts that can be applied in situations that involve cellulosic materials. Indeed, the literature citations show that a great deal of work has been carried out related to permeability loss phenomena; however only a minority of the studies have involved fibrous filter media, let alone cellulosic filters. Few published studies have considered effects related to the shapes of suspended particles, and effects of elongated suspended particles are hardly mentioned in the general literature related to permeability loss, except in the case of articles specifically dealing with paper manufacturing issues.

The situation just described presents two kinds of opportunities for those who are especially interested in cellulosic materials. One set of opportunities lies in the adoption and use of findings that were originally developed with non-cellulosic filter media or particulate matter in mind. Judgment will be needed to make sure that the assumptions inherent in some such approaches are not too divergent so as to render them non-useful from the standpoint of a cellulosic technologist. In other cases, such as when applying concepts of membrane fouling to the use of cellulosic membranes, there is an obvious fit between certain past work and likely applications involving membranes prepared from cellulosic materials.

Another set of opportunities lies in the area of scholarly research. There is a clear need for studies involving flow through fibrous media, showing how permeability loss phenomena can be related to such factors as particle size and shape. Tests can be carried out to determine whether there are useful power-law relationships between amounts of captured particles and net permeability through fibrous filter media. Analyses can be carried out with deterministic or stochastic approaches, aimed at verifying whether the same kinds of pore network models, which have proven their worth in packed bed applications, also can be applied to the process of forming a sheet of paper, for instance. Advances in our understanding of hydrodynamic interactions, colloidal forces between surfaces in aqueous environments, and in computational power will make it possible to conduct theoretical studies that would not have been feasible in the past.

\section{ACKNOWLEDGMENTS}

The authors wish to thank James E. Amburgey (University of North Carolina, Charlotte) and Bandaru V. Ramarao (State University of New York, College of Environmental Science and Forestry) for their many helpful suggestions.

\section{REFERENCES CITED}

Al-Abduwani, F. A. H., Shirzadi, A., van den Broek, W. M. G. T., and Currie, P. K. (2003). "Formation damage vs. solid particles deposition profile during laboratory simulated,” PWRI. SPE-82235. European Formation Damage Conference, The Hague, The Netherlands, 13-14 May 2003. 
Alince, B., and Lepoutre, P. (1983). "Viscosity, packing density, and optical properties of pigment blends,” Colloids Surf. 6(2), 155-165.

Alvarez, A. C., Hime, G., Marchesin, D., and Bedrikovetsky, P. G. (2007). “The inverse problem of determining the filtration function and permeability reduction in flow of water with particles in porous media,” Transport in Porous Media 70(1), 43-62.

Amburgey, J. E. (2005). "Optimization of the extended terminal subfluidization wash (ETSW) filter backwashing procedure,” Water Research 39(2-3), 314-330.

Amburgey, J. E., and Amirtharajah, A. (2005). "Strategic filter backwashing techniques and resulting particle passage,” J. Environ. Eng. - ASCE 131(4), 535-547.

Aydin, B., Bilodeau, S., Hamidipour, M., Larachi, F., and Kleitz, F. (2008). "Polymerfilled composite porous catalytic particles for hydrodynamic studies in trickle-bed reactors,” Ind. Eng. Chem. Res. 47(8), 2569-2578.

Bai, R., and Tien, C. (2000). "Transient behavior of particle deposition in granular media under various surface interactions,” Colloids Surf. A 165(1-3), 95-114.

Bai, R., and Tien, C. (1997). "Particle detachment in deep bed filtration,” J. Colloid Interface Sci. 186(2), 307-317.

Barrande, M., Bouchet, R., and Denoyel, R. (2007). “Tortuosity of porous particles,” Anal. Chem. 79(23), 9115-9121.

Bauer, D., Goyeau, B., and Gobin, D. (2008). "Large particle transport in porous media: Effect of pore plugging on the macroscopic transport properties,” J. Porous Media 11(4), 343-360.

Bedrikovetsky, P., Marchesin, D., Checaira, F., Serra, A. L., and Resende, E . (2001). "Characterization of deep bed filtration system from laboratory pressure drop measurements,” J. Petroleum Sci. Eng. 32(2-4), 167-177.

Belfort, G., Davis, R. H., and Zydney, A. L. (1994). "The behaviour of suspensions and macromolecular solutions in crossflow microfiltration,” J. Memb. Sci. 96, 1-58.

Belfort, G., Pimbley, J. M., Greiner, A., and Chung, K. Y. (1993). "Diagnosis of membrane fouling using a rotating annular filter. 1. Cell culture media,” J. Membrane Sci. 77, 1-22.

Berube, P. R., and Lei, E. (2006). “The effect of hydrodynamic conditions and system configurations on the permeate flux in a submerged hollow fiber membrane system," J. Membrane Sci. 271(1-2), 29-37.

Bessiere, Y., Fletcher, D. F., and Bacchin, P. (2008). "Numerical simulation of colloid dead-end filtration: Effect of membrane characteristics and operating conditions on matter accumulation,” J. Membrane Sci. 313(1-2), 52-59.

Binotto, A. P., and Nicholls, G. A. (1979). "Correlation of morphological variations in pulps with filtration resistance, specific volume, and specific surface,” Appita 33 (3), 185-188 (1979).

Boerlage, S. F. E., Kennedy, M. D., Dickson, M. R., El-Hodali, D. E. Y., and Schippers, J. C. (2002). "The modified fouling index using ultrafiltration membranes (MFIUF): Characterization, filtration mechanisms and proposed reference membrane,” $J$. Membrane Sci. 197, 1-21.

Boller, M. A., and Kavanaugh, M. C. (1995). "Particle characteristics and headloss increase in granular media filtration,” Water Res. 29(4), 1139-1149. 
Bolton, G., LaCasse, D., and Kuriyel, R. (2006). “Combined models of membrane fouling: Development and application to microfiltration and ultrafiltration of biological fluids,” J. Membrane Sci. 277, 75-84.

Bolton, G. R., LaCasse, D., Lazzara, M. J., and Kuriyel, R. (2005). “The fiber-coating model of biopharmaceutical depth filtration,” AIChE J. 51(11), 2978-2987.

Bowen, W. R., Calvo, J. I., and Hernández, A. (1995). "Steps of membrane blocking in flux decline during protein microfiltration,” J. Membrane Sci. 101, 153-165.

Bradford, S. A., Bettahar, M., Simunek, J., and van Genuchten, M. Th. (2004). "Straining and attachment of colloids in physically heterogeneous porous media,” Vadose Zone J. 3, 384-394.

Bradford, S. A., Simunek, J., Bettahar, M., Genuchten, M. T., and Van Yates, S. R. (2003). "Modeling colloid attachment, straining, and exclusion in saturated porous media,” Environ. Sci. Technol. 37(10), 2242-2250.

Brecht, W., and Klemm, K. (1953). "The mixture of structures in a mechanical pulp as a key to the knowledge of its technical properties,” Pulp Paper Mag. Can. 54(1), 72-80.

Brenner, H. (1968). "The slow motion of a sphere through a viscous fluid towards a plane surface,” Chem. Eng. Sci. 16(3-4), 242-251.

Britt, K. W., Unbehend, J. E., and Shridharan, R. (1986). "Observations on water removal in papermaking,” Tappi J. 69(7), 76-79 (1986).

Bryant, S. L., and Blunt, M. (1992). "Prediction of relative permeability in simple porous media,” Phys. Rev. A 46(4), 2004-2011.

Bryant, S. L., Mellor, D. W., and Cade, C. A. (1993). "Physically representative network models of transport in porous media," AIChE J. 39, 387-396.

Burganos, V. N., Paraskeva, C. A., and Payatakes, A. C. (1992). “Three-dimensional trajectory analysis and network simulation of deep bed filtration,” J. Colloid Interface Sci. 148(1), 167-181.

Burganos, V. N., Skouras, E. D., Parakeva, C. A., and Payatakes, A. C. (2001). "Simulation of the dynamics of depth filtration of non-Brownian particles," AIChE J. 47(4), 880-894.

Carman, P. C. (1937). “Fluid flow through granular beds,” Trans. I. Chem. E. 15, 150166.

Carman, P. C. (1938). "The determination of the specific surface of powders. I.,” Soc. Chem. Industry (Trans. \& Communications) 57, 225-234.

Carrier, W. D. (2002). “Goodby, Hazen; Hello, Kozeny-Carman,” J. Geotech. Geoenviron. Eng. (Nov.), 1054-1056.

Carroll, T. (2001). "The effect of cake and fibre properties on flux declines in hollowfibre microfiltration membranes,” J. Membrane Sci. 189(2), 167-178.

Cerda, C. M. (1987a). "Mobilization of quartz fines in porous media,” Clays Clay Minerals 36, 491-497.

Cerda, C. M. (1987b). “Mobilization of kaolinite fines in porous media,” Colloids Surf. 27, 219-241.

Chapuis, R. P., and Auberton, M. (2003). "On the use of the Kozeny-Carman equation to predict the hydraulic conductivity of soils,” Can. J. Geotech. 40, 616-628.

Chiang, H. W., and Tien, C. (1985). "Dynamics of deep bed filtration. 1. Analysis of two limiting situations,” AIChE J. 31(8), 1349-1359. 
Chidaglia, C., de Arcangelis, L., Hinch, J., and Guazzelli, E. (1996b). "Hydrodynamic interactions in deep bed filtration,” Phys. Fluids 8(1), 6-14.

Choo, C.-U., and Tien, C. (1995a). “Analysis of transient behavior of deep bed filtration,” J. Colloid Interface Sci. 169(1), 13-33.

Choo, C.-U., and Tien, C. (1995b). "Simulation of hydrosol deposition in granular media,” AIChE J. 41(6), 1426-1442.

Clague, D. S., Kandhai, B. D., Zhang, R., and Sloot, P. M. A. (2000). "Hydraulic permeability of (un)bounded fibrous media using the lattice Boltzmann method," Phys. Rev. E. 61, 616-625.

Cleasby, J. L., Williamson, M. M., Baumann, E. R. (1963). "Effect of filtration rate changes on quality,” J. Amer. Water Works Assoc. 55, 869-877.

Cole, C. A., Hubbe, M. A., and Heitmann, J. A. (2008). "Water release from fractionated stock suspensions. 1. Effects of the amounts and types of fiber fines,” TAPPI J. 7(7), 28-32.

Curry, R. B., and Beasley, R. P. (1962). "Flow of colloidal suspensions through porous media as related to reservoir sealing," Trans. ASAE 4(2), 160-164.

Darby, J. L., and Lawler, D. F. (1990). "Ripening in depth filtration - Effect of particle size on removal and head loss," Environ. Sci. Technol. 24(7), 1069-1079.

Datta, S., and Redner, S. (1998). “Gradient clogging in depth filtration,” Phys. Rev. E 58(2), R1203-R1206.

Davis, R. H. (1992). "Modeling of fouling of cross-flow microfiltration membranes," Separation Purification Methods 21(2), 75-126.

De, D., Hrymak, A. N., and Pelton, R. (1997). "Polyelectrolyte precipitate formation during miscible displacement in porous media,” AIChE J. 43(10), 2415-2423.

De Bruijn, J. P. F., Salazar, F. N., and Borquez, R. (2005). "Membrane blocking in ultrafiltration - A new approach to fouling,” Tans. I. Chem. E, Part C: Food Bioprod. Proc. 83(C3), 211-219.

Duclos-Orsello, C., Li, W., and Ho, C. C. (2006). “A three mechanism model to describe fouling of microfiltration membranes,” J. Membr. Sci. 280(1-2), 856-866.

Dullien, F. A. L. (1975). "New network permeability model of porous media," AIChE J. 21(2), 299-307.

Dunleavey-Routh, R., and Vincent, B. (2007). “Aggregate morphology and aggregation rate constants for silica dispersions in the presence of salt and polyelectrolyte," $J$. Colloid Interface Sci. 309(1), 119-125.

Durmusoglu, E., and Corapcioglu, M. Y. (2000). "Experimental study of horizontal barrier formation by colloidal silica,” J. Environ. Eng. - ASCE 126(9), 833-841.

Elimelech, G., Gregory, J., Jia, X., and Williams, R. A. (1995). Particle Deposition and Aggregation, Butterworth-Heinemann, USA.

Faibish, R. S., Elimelech, M., and Cohenn, Y. (1998). "Effect of interparticle electrostatic double layer interactions on permeate flux decline in crossflow membrane filtration of colloidal suspensions: An experimental investigation,” J. Colloid Interface Sci. 204, 77-86.

Fan, L. T., Hwang, S. H., Nassar, R., and Chou, S. T. (1985a). "An experimental study of deep-bed filtration: Stochastic analysis,” Powder Technol. 44(1), 1-11. 
Fan, L. T., Nassar, R., Hwang, S. H. and Chou, S. T. (1985b). “Analysis of deep bed filtration data: Modeling as a birth-death process,” AIChE J. 31(11), 1781-1790.

Fatt, I. (1956). “The network model of porous media: I, II, III,” Pet. Trans. AIME 207, 144-188.

Fitzpatrick, C. S. B. (1993). “Observations of particle detachment during filter backwashing,” Water Sci. Technol. 27(10), 213-221.

Fu, L. F., and Dempsey, B. A. (1998). "Modeling the effect of particle size and charge on the structure of the filter cake in ultrafiltration,” J. Membrane Sci. 149(2), 221-240.

Gabriel, G. A., and Inamdar, G. R. (1983). “An experimental investigation of fines migration in porous media,” $58^{\text {th }}$ SPE Ann. Meeting, San Francisco, Oct., Paper No. 12168.

Ganter, C. G., Jakob, S. M., and Takala, J. (2006). "Pulmonary capillary pressure - A review.” Minerva Anestesiologica 72(1-2), 21-36.

Ginn, T. M., Amirtharajah, A., and Karr, P. R. (1992). "Effects of particle detachment in granular-media filtration,” J. Amer. Water Works Assoc. 84(2), 66-76.

Gironès, M., Lammertink, R. G. H., and Wessling, M. (2006). "Protein aggregate deposition and fouling reduction strategies with high-flux silicon nitride microsieves,” J. Membrane Sci. 273, 68-76.

Goldenberg, L. C. (1985). "Decrease of hydraulic conductivity in sand at the interface between seawater and dilute clay suspensions,” J. Hydrology 78(1-2), 183-199.

Goren, S. L., and O’Neill, M. E. (1971). "On the hydrodynamic resistance to a particle of a dilute suspension when in the neighborhood of a large obstacle," Chem. Eng. Sci. 26(3), 325-338.

Grace, H. P. (1956). “Structure and performance of filter media,” AIChE J. 2, 307-336.

Gray, M. R., Srinivasan, N., and Masliyah, J. H. (2002). "Pressure buildup in gas-liquid flow through packed beds due to deposition of fine particles,” Can. J. Chem. Eng. 80(3), 346-354.

Gruesbeck, C., and Collins, R. E. (1982). "Entrainment and deposition of fine particles in porous media," SPE J. 847-856.

Hamdi, N., and Srasra, E. (2008). "Filtration properties of two Tunisian clays suspensions: Effect of the nature of clay,” Desalination 220(1-3), 194-199.

Hampton, J. H. D., Savage, S. B., and Drew, R. A. L. (1993). “Computer modeling of filter pressing and clogging in a random tube network,” Chem. Eng. Sci. 48(9), 16011611.

Han, S. T. (1969). “Compressibility and permeability of fiber mats,” Pulp Paper Mag. Can. 70(9), T134-T146, 65-77.

Hardcastle, J. H., and Mitchel, J. K. (1974). "Electrolyte concentration permeability relationships in sodium illite silt mixtures,” Clays Clay Minerals 22, 143-154.

Heertjes, P. M. (1957). "Studies in filtration: Blocking filtration,” Chem Eng Sci. 6(4-5), 190-203.

Hermans, P. H., and Bredée, H. L. (1936). "Principles of the mathematical treatment of constant-pressure filtration,” J. Soc. Chem. Ind. 55T, 1-4.

Herroro, C. , Prádanos, P., Calvo, J. I., Tejerina, F., and Hernández, A. (1997). "Flux decline in protein microfiltration: Influence of operative parameters,” J. Colloid Interface Sci. 187(2), 344-351. 
Herzig, J. P., Leclerc, D. M., and le Goff, P. (1970). "Flow of suspensions through porous media - Application to deep filtration,” Industrial Eng. Chem. 62(5), 8-35.

Hiemenz, P. C., and Rajagopalan, R. (1997). Principles of Colloid and Surface Science, $3^{\text {rd }}$ Ed., Dekker, New York.

Higdon, J. J. L., and Ford, G. D. (1996). "Permeability of three-dimensional models of fibrous porous media,” J. Fluid Mech. 308, 341-361.

Ho, C. C., and Zydney, A. L. (2000). “A combined pore blockage and cake filtration model for protein fouling during microfiltration,” J. Colloid Interface Sci. 232, 389399.

Howe, K. J., and Clark, M. M. (2002). "Fouling of microfiltration and ultrafiltration membranes by natural waters,” Environ. Sci. Technol. 36, 3571-3576.

Huang, C. H., Chang, C. L., and Lin, T. S. (2007), "Classification of particles in particleladen stream through a stainless steel fibrous filter,” Korean J. Chem. Eng. 24(3), 413-417.

Hubbe, M. A. (1985). "Detachment of colloidal hydrous oxide spheres from flat solids exposed to flow. 2. Mechanism of release," Colloids and Surfaces 16(3-4), 249-270.

Hubbe, M. A. (2002). "Fines management for increased paper machine productivity," Proc. Sci. Tech. Advan. Wet End Chem., PIRA, Vienna, paper 3.

Hubbe, M. A., and Heitmann, J. A. (2007). "Review of factors affecting the release of water from cellulosic fibers during paper manufacture,” BioRes. 2(3), 500-533.

Hubbe, M. A., Heitmann, J. A., and Cole, C. A. (2008). "Water release from fractionated stock suspensions. 2. Effects of consistency, flocculants, shear, and order of mixing,” TAPPI J. 7(8), 14-19.

Hubbe, M. A., Rojas, O. J., Lee, S. Y., Park, S., and Wang, Y. (2007). “Distinctive electrokinetic behavior of nanoporous silica particles treated with cationic polyelectrolyte,” Colloids and Surfaces A 292(2), 271-278.

Iliuta, I., and Larachi, F. (2003). "Fines deposition dynamics in packed-bed bubble reactors,” Indust. Eng. Chem. Res. 42(12), 2441-2449.

Iliuta, I., and Larachi, F. (2005). "Modeling simultaneous biological clogging and physical plugging in trickle-bed bioreactors for wastewater treatment," Chem. Eng. Sci. 60(5), 1477-1489.

Iliuta, I., and Larachi, F. (2006). "Deposition and aggregation of Brownian particles in trickle-bed reactors,” AIChE J. 52(12), 4167-4180.

Imdakm, A. O., and Sahimi, M. (1987). "Transport of large particles in flow through porous media,” Phys. Rev. A 36, 5304-5309.

Imdakm, A. O., and Sahami, M. (1991). "Computer simulation of particle transport in flow through porous media,” Chem. Eng. Sci. 46(8), 1977-1993.

Ingmanson, W. L. (1952). "An investigation of the mechanisms of water removal from pulp slurries,” Tappi 35(10), 439-448.

Ingmanson, W. L. (1953). "Filtration resistance of compressible materials,” Chem. Eng. Progr. 49(11), 577-584.

Ioannidis, M. A., and Chatzis, I. (2000). "On the geometry and topology of 3D stochastic porous media,” J. Colloid Interface Sci. 229(2), 323-334. 
Ioannidis, M. A., Kwiecien, M. J., and Chatzis, I. (1997). "Electrical conductivity and percolation aspects of statistically homogeneous porous media,” Transp. Porous Media 29(1), 61-83.

Iritani, E., Mukai, Y., Tanaka, Y., and Murase, T. (1995). "Flux decline behavior in deadend microfiltration of protein solutions,” J. Membrane Sci. 103(1-2), 181-191.

Iritani, E., Katagiri, N., Sugiyama, Y., and Yagishita, K. (2007). “Analysis of flux decline behaviors in filtration of very dilute suspensions,” AIChE J. 53(9), 2275-2283.

Ishizaki, K., Komarneni, S., and Nanko, M. (1998). Porous Materials: Process Technology and Applications, Materials Technol. Ser., Kluwer Acad. Publ., 240 pp.

Ison, C. R., and Ives, K. J. (1969). "Removal mechanisms in deep bed filtration,” Chem. Eng. Sci. 24(4), 717-729.

Ives, K. J., and Pienvichitr, V. (1965). "Kinetics of the filtration of dilute suspensions," Chem. Eng. Sci. 20(11), 965-973.

Iwasaki, T. (1937). “Some notes on sand filtration,” J. Am. Water Works Ass. 29(10), 1591-1602.

Jackson, G. W., and James, D. F. (1986). “The permeability of fibrous porous media,” Can. J. Chem. Eng. 64, 364-374.

Japuntich, D. A., Stenhouse, J. I. T., and Liu, B. Y. H. (1994). "Experimental results of solid monodisperse particle clogging of fibrous filters," J. Aerosol Sci. 25(2), 385393.

Jegatheesan, V., Vigneswaran, S., and Lee, S. H. (2005). “Deposition of submicron particles in deep bed filtration under unfavorable surface conditions,” Korean J. Chem. Eng. 22(1), 142-146.

Jiang, T., Kennedy, M. D., van der Meer, W. G. J., Vanrolleghem, P. A., and Schippers, J. C. (2003). "The role of blocking and cake filtration in MIBR fouling," Desalination 157(1-3), 335-343.

Jimenez-Gonzalez, I., Rodriguez-Navarro, C., and Scherer, G. W. (2008). "Role of clay minerals in the physicomechanical deterioration of sandstone,” J. Geophys. Res. Earth Sci. 113(F2), article no. F02021.

Johnson, P. R., and Elimelech, M. (1995). "Dynamics of colloid deposition in porousmedia - Blocking based on random sequential adsorption,” Langmuir 11(3), 8701812.

Johnson, W. P., Li, X. Q., and Yal, G. (2007). "Colloid retention in porous media: Mechanistic confirmation of wedging and retention in zones of flow stagnation,” Environ. Sci. Technol. 41(4), 1279-1287.

Johnston, N., and Beeson, C. M. (1945). "Water permeability of reservoir sands,” Trans. AIME 160, 43-55.

Jones, F. O. (1964). "Influence of chemical composition of water on clay blocking of permeability,” Trans. AIME 231, 441-446.

Kampel, G., Goldsztein, G. H., and Santamarina, J. C. (2008). "Plugging of porous media and filters: Maximum clogged porosity,” Appl. Phys. Let. 92(8), article 084101.

Katsoufidou, K., Yiantsios, S. G., and Karabelas, A. J. (2008). “An experimental study of UF membrane fouling by humic acid and sodium alginate solutions: The effect of backwashing on flux recovery,” Desalination 220(1-3), 214-227. 
Kau, S. M., Lawler, D. F. (1995). “Dynamics of deep-bed filtration: Velocity, depth and media,” J. Environ. Eng. 121(12), 850-859.

Khilar, K. C. (1983). "Effect of streaming potential on permeability of sandstones,” Ind. Eng. Chem. Fundam. 22, 264-266.

Khilar, K. C., and Fogler, H. S. (1983). “Water sensitivity of sandstones,” SPE J. 23(1), 55-64.

Khilar, K. C., and Fogler, H. S. (1984). "The existence of a critical salt concentration for particle release,” J. Colloid Interface Sci. 101(1), 214-224.

Khilar, K. C., and Fogler, H. S. (1987). "Colloidally induced fines migration in porous media,” Rev. in Chem. Eng. 4(1-2), 41-108.

Khilar, K., and Fogler, S. (1998). Migration of Fines in Porous Media, Kluwer Academic Publishers, Dordrecht/London/Boston.

Khilar, K. C., Fogler, H. S., and Ahlavalia, J. S. (1983). "Sandstone water sensitivity: Existence of a critical rate of salinity decrease for particle capture,” Chem. Eng. Sci. 38, 789-809.

Kilduff, J. E., Mattaraj, S., Zhou, M. Y., Belfort, G. (2005). “Kinetics of membrane flux decline: The role of natural colloids and mitigation via membrane surface modification,” J. Nanopartice Res. 7(4-5), 525-544.

Kim, K. J., Chen, V., and Fane, A. G. (1993). "Ultrafiltration of colloidal silver particles: Flux, rejection, and fouling,” J. Colloid Interface Sci. 155, 347-359.

Kim, Y. S., and Whittle, A. J. (2006). "Filtration in a porous granular medium: 2. Application of bubble model to 1-D column experiments," Transp. Porous Media 65(2), 309-335.

Kozeny, J. (1927). “About capillaries conducting water in the earth,” Sitzber. Akd. Wiss. Wien, Math. Naurv. Kasse 136(Abl. 2a), 271-306.

Krogerus, B., Fagerholm, K., and Tiikkaja, E. (2002). "Fines from different pulps compared by image analysis,” Nordic Pulp Paper Res. J. 17(4), 440-444.

Kruger, R. F. (1986). "An overview of formation damage and well productivity in oilfield operations,” J. Pet. Technol. (Feb.), 131-152.

Kuhnen, F., Barmettler, K., Bhattacharjee, S., Elimelech, M., and Kretzschmar, R. (2000). “Transport of iron oxide colloids in packed quartz sand media: Monolayer and multilayer deposition,” J. Colloid Interface Sci. 231, 32-41.

Kulkarni, P., Sureshkumar, R., and Biswas, P. (2005). "Hierarchical approach to model multilayer colloidal deposition in porous media,” Environ. Sci. Technol. 39(17), 6361-6370.

Kumar, P., Wei, H. L., and Ramarao, B. V. (1996). “A model for freeness measurement of papermaking suspensions,” Chem. Eng. Commun. 1996, 152-153, 287-306.

Larson, T. M., Davis, J., Lam, H., and Cacia, J. (2003). "Use of process data to assess chromatographic performance in productionscale protein purification columns," Biotechnol. Prog. 19(2), 485-492.

Le Coq, L. (2008). "Influence on permeability of the structural parameters of heterogeneous porous media,” Environmental Technol. 29(2), 141-149.

Le Coq, L., and Silvy, J. (1999). "Fibrous media plugging modeling for liquid filtration," Chem. Eng. Commun. 174, 145-166. 
Lee, J., and Koplik, J. (2001). “Network model for deep bed filtration,” Phys. Fluids 13(5), 1076-1078.

Liimatainen, H., Haavisto, S., Haapala, A., and Niinimäki, J. (2009). "Influence of adsorbed and dissolved carboxymethyl cellulose on fibre susepnsion dispersing, dewaterability, and fines retention,” BioResources 4(1), 321-340.

Linden, T., Ljunglof, A., Kula, M.-R., and Thommes, J. (1999). "Visualizing twocomponent protein diffusion in porous adsorbents by confocal scanning laser microscopy,” Biotechnol. Bioeng. 65(6), 622-630.

Liu, X. A., Whiting, P., Pande, H., and Roy, D. N. (2001). “The contribution of different fractions of fines to pulp drainage in mechanical pulps,” J. Pulp Paper Sci. 27(4), 139-143.

Maa, Y.-F., and Hsu, C. C. (1998). "Investigation of fouling mechanisms for recombinant human growth hormone sterile filtration,” J. Pharm. Sci. 87, 808-812.

Mackie, R. I., Homer, R. M. W., and Jarvis, R. J. (1987). “Dynamic modeling of deep bed filtration,” AIChE J. 33(11), 1761-1775.

Madaeni, S. S. (1997). “The ultrafiltration of very dilute colloidal gold suspensions,” J. Porous Mater. 4, 31-44.

Maroudas, A., and Eisenklam, P. (1965a). "Clarification of suspensions: A study of particle deposition in granular media: I. Some observations on particle deposition," Chem. Eng. Sci. 20(10), 867-873.

Maroudas, A., and Eisenklam, P. (1965b). "Clarification of suspensions: A study of particle deposition in granular media: 11. A Theory of clarification,”, Chem. Eng. Sci. 20(10), 875-888.

Matsumoto, K., Hirata, S, and Ohya, H. (1992). “Microfiltration model considering incomplete pore blocking and incomplete capture of particles by cake,” Kagaku Kogaku Ronbunshu 18(4), 455-462.

Mavredaki, E., Neofotistou, E., and Demadis, K. D. (2005). "Inhibition and dissolution as dual mitigation approaches for colloidal silica fouling and deposition in process water systems: Functional synergies,” Ind. Eng. Chem. Res. 44, 7019-7026.

Mays, D. C., and Hunt, J. R. (2005). "Hydrodynamic aspects of particle clogging in porous media,” Environ. Sci. Technol. 39(2), 577-584.

McCabe. W. L., Smith, J. C., and Harriott, P. (2005). Unit Operations in Chemical Engineering, 7th Ed., McGraw-Hill, Boston.

McDonough, R. M., Welsch, K., Fane, A. G., and Fell, C. J. D. (1992). “Incorporation of the cake pressure profiles in the calculation of the effect of particle charge on the permeability of filter cakes obtained in the filtration of colloids and particulates,” $\mathrm{J}$. Membrane Sci. 72(2), 197-204.

McDowell-Boyer, L. M., Hunt, J. R., and Sitar, N. (1986). "Particle transport through porous media,” Water Resour. Res. 22(13), 1901-1921.

Meng, F. G., Zhang, H. M., Li, Y. S., Zhang, X. W., and Yang, F. L. (2005). "Application of fractal permeation model to investigate membrane fouling in membrane bioreactor," J. Membrane Sci. 262(1-2), 107-116.

Meyer, H. (1962). "A filtration theory of compressible fibrous beds formed from dilute suspensions,” Tappi 45(4), 296-310. 
Mishra, A. K., Ohtsubo, M., Li, L., and Higashi, T. (2005). "Effect of salt concentrations on the permeability and compressibility of soil-bentonite mixtures," J. Faculty Agriculture Kyushu Univ. 50(2), 837-849.

Mohan, K. K., and Fogler, H. S. (1997). "Effect of pH and layer charge on formation damage in porous media containing swelling clays,” Langmuir 13(10), 2863-2872.

Mojarad, R. S., and Settari, A. (2007). "Coupled numerical modeling of reservoir flow with formation plugging,” J. Canadian Petroleum Technol. 46(3), 54-59.

Monaghan, P. H., Salatheil, R. E., and Morgann, B. E. (1959). "Laboratory studies of formation damage in sands containing clays,” J. Pet. Technol. Trans. AIME 11(2), 209-215.

Mourouzidis-Mourouzis, S. A., and Karabelas, A. J. (2006). "Whey protein fouling of microfiltration ceramic membranes - Pressure effects,” J. Membrane Sci. 282(1-2), 124-132.

Muecke, T. W. (1979). "Formation fines and factors controlling their movement in porous media,” J. Pet. Technol. (Feb.), 144-150.

Mungan, N. (1965). "Permeability reduction through changes in $\mathrm{pH}$ and salinity," J. Pet. Technol. 1449-1453.

Murase, T., Ohn, T., and Kimata, K. (1995). "Filtrate flux in cross-flow microfiltration of dilute suspension forming a highly compressible fouling cake layer,” J. Membrane Sci. 108(1-2), 121-128.

Narayan, R., Coury, J. R., Masliyah, J. H., and Gray, M. R. (1997). "Particle capture and plugging in packed-bed reactors,” Indust. Eng. Chem. Res. 36(11), 4620-4627.

Nascimento, A. G., Totola, M. R., Souza, C. S., Borges, M. T., and Borges, A. C. (2006). "Temporal and spatial dynamics of blocking and ripening effects on bacterial transport through a porous system: A possible explanation for CFT deviation," Colloids Surf. B. - Biointerfaces 53(2), 241-244.

O’Melia, C. R., and Ali, W. (1978). "The role of retained particles in deep bed filtration," Prog. Water Technol. 10, 167-182.

Pandya, V. B., Bhuniya, S., and Khilar, K. C. (1998). "Existence of a critical particle concentration in plugging of a packed bed,” AIChE J. 44(4), 978-981.

Paradis, M. A., Genco, J. M., Bousfield, D. W., Hassler, J. C., and Wildfong, V. (2002). "Determination of drainage resistance coefficients under known shear rate," Tappi J. $1(8), 12-18$.

Parry, A. J. (2006). "Numerical prediction method for growth and deformation of filter cakes,” J. Fluids Eng. - Trans ASME 128(6), 1259-1265.

Patel, M., and Trivedi, R. (1994). "Variations in strength and bonding properties of fines from filler, fiber, and their aggregates,” Tappi J. 77(3), 185-192.

Payatakes, A. C., Park, H. Y., and Petrie, J. (1981). "A visual study of particle deposition and reentrainment during depth filtration of hydrosols with polyelectrolyte," Chem. Eng. Sci. 36(8), 1319-1335.

Payatakes, A .C., Tien, C., and Turian, R. M. (1973). “A new model for granular porous media. I. Model formulation,” AIChE J. 19(1), 58-76.

Pensdse, H., Tien, C. and Turian, R. M. (1978). "Dispersion measurement in clogged filter beds - A diagnostic study on morphology of particle deposits," AIChE J. 24(3), 473-485. 
Philipse, A. P., and Wierenga, A. M. (1998). “On the density and structure formation in gels and clusters of colloidal rods and fibers,” Langmuir 14(1), 49-54.

Przybysz, K., and Szwarcsztajn, E. (1973). “Effect of crill on pulp freeness,” Przeglad Papier 29(4), 105-108.

Purkait, M. K., Bhattacharya, P. K., and De, S., (2005). "Membrane filtration of leather plant effluent: Flux decline mechanism,” J. Membr. Sci. 258(1-2), 85-96.

Putnam, D. D., and Burns, M. A. (1997). "Predicting the filtration of non-coagulating particles in depth filters,” Chem. Eng. Sci. 52(1), 93-105.

Räisänen, K. (1996). "High-vacuum dewatering on a paper machine wire section - A literature review,” Paperi Puu 78(3), 113-120.

Ramachandran, V., Khilar, K. C., and Fogler, H. S. (1998). "Use of network models for prediction of permeability reduction due to fines entrapment,” in Khilar, K., and Fogler, S. (1998). Migration of Fines in Porous Media, Kluwer Academic Publishers, Dordrecht/London/Boston.

Rausch, D. L., and Curry, R. B. (1963). "Effect of viscosity and zeta potential of bentonite suspensions on flow through porous media,” Trans. ASAE 6, 167-169.

Raveendran, P., and Amirtharajah, A. (1995). "Role of short-range forces in particle detachment during filber backwashing,” J. Environ. Eng. - ASCE 121(12), 860-868.

Reed, M. G. (1980). "Gravel pack and formation sandstone dissolution during steam injection,” J. Pet. Tech. 32, 941-949.

Rege, S. D., and Fogler, H. S. (1987). "Network model for straining dominated particle entrapment in porous media,” Chem. Eng. Sci. 42(7), 1553-1564.

Rege, S. D., and Fogler, H. S. (1988). “A network model for deep bed filtration of solid particles and emulsion drops,” AIChE J. 34(14), 1761-1772.

Rijnaarts, H. H. M., Norde, W., Bouwer, E. J., Lyklema, J., and Zehnder, A. J. B. (1996). "Bacterial deposition in porous media related to the clean bed collision efficiency and to substratum blocking by attached cells,” Environ. Sci. Technol. 30(10), 2869-2876.

Ryan, J. N., and Elimelech, M. (1996). "Colloid mobilization and transport in groundwater,” Colloids Surf. A 107, 1-56.

Saffman, P. G. (1965). “The lift on a small sphere in a slow shear flow,” J. Fluid Mech. 22(2), 385-400.

Sahimi, M., Gavalas, G. R., and Tsotsis, T. T. (1990). "Statistical and continuum models of fluid-solid reactions in porous media,” Chem. Eng. Sci. 45(6), 1443-1502.

Sakthivadivel, R., Thanikachalam, V., and Seetharaman, S. (1972). "Head-loss theories Inflitration,” J. Amer. Water Works Assoc. 8, 233-238.

Santos, A., Bedrikovetsky, P., and Fontoura, S. (2008). “Analytical micro model for size exclusion: Pore blocking and permeability reduction,” J. Membrane Sci. 308(1-2), 115-127.

Seminario, L., Rozas, R., Borquez, R., and Toledo, P. G. (2002). "Pore blocking and permeability reduction in cross-flow microfiltration,” J. Membrane Sci. 209(1), 121142.

Shapiro, A. A., Bedrikovetsky, P. G., Santos, A., and Medvedev, O. O. (2007). “A stochastic model for filtration of particulate suspensions with incomplete pore plugging,” Transport Porous Media 67(1), 135-164. 
Sharma, M. M., Kuo, J. F., and Yen, T. F. (1987). "Further investigation of the surface charge properties of oxide surfaces in oil-bearing sands and sandstones,” J. Colloid Interface Sci. 115(1), 9-16.

Sharma, M. M., and Yortsos, Y. C. (1987a). "Transport of particulate suspensions in porous media: model formulation,” AIChE J. 33(10), 1636-1643.

Sharma, M. M., and Yortsos, Y. C. (1987b). “A network model for deep bed filtration processes,” AIChE J. 33(10), 1644-1653.

Sharma, M. M., and Yortsos, Y. C. (1987c). “Fines migration in porous media,” AIChE J. 33(10), 1654-1662.

Simunek, J., He, C. M., Pang, L. P., and Bradford, S. A. (2006). “Colloid-facilitated solute transport in variably saturated porous media: Numerical model and experimental verification,” Vadose Zone J. 5(3), 1035-1047.

Sin, S. C., Baldascini, H., Hearle, D. C., Hoare, M., and Titchener-Hooker, N. J. (2006). "Effect of fouling on the capacity and breakthrough characteristics of a packed bed ion exchange chromatography column,” Bioproc. Biosys. Eng. 28(6), 405-414.

Singh, G., and Song, L. (2005). "Quantifying the effect of ionic strength on colloidal fouling potential in membrane filtration,” J. Colloid Interface Sci. 284(2), 630-638.

Singh, G., and Song, L. F. (2006). "Cake compressibility of silica colloids in membrane filtration processes,” Indust. Eng. Chem. Res. 45(22), 7633-7638.

Singh, R., Lavrykov, S., and Ramarao, B. V. (2009). "Permeability of pulp fiber mats with filler particles,” Colloids Surf. A 333(1-3), 96-107.

Siu, S. C., Boushaba, R., Liau, J., Hjorth, R., and Titchener-Hooker, N. J. (2007). "Confocal imaging of chromatographic fouling under flow conditions," J. Chem. Technol. Biotechnol. 82(10), 871-881.

Skerlos, S. J., Rajagopalan, N., DeVor, R. E., Kapoor, S. G., and Angspatt, V. D. (2001). "Microfiltration of polyoxyalkylene metalworking fluid additives using aluminum oxide membranes,” J. Manuf. Sci. Eng., Trans. ASME 123(4), 692-699.

Smellie, R. H., and La Mer, V. K. (1958). "Flocculation, subsidence and filtration of phosphate slimes. VI. A quantitative theory of filtration of flocculated suspensions," J. Colloid Interface Sci. 23(3), 589-599.

Song, L., and Singh, G. (2005). "Influence of various monovalent cations and calcium ions on the colloidal fouling potential,” J. Colloid Interface Sci. 289(2), 479-487.

Staby, A., Johansen, N., Wahlstrom, H., and Mollerup, I. (1998). “Comparison of loading capacities of various proteins and peptides in culture medium and in pure state,” $J$. Chromatogr. A 827(2), 311-318.

Steenberg, B., Sandgren, B., and Wahren, D. (1960). “Studies on pulp crill,” Svensk Papperstidn. 63(12), 395-397.

Tanaka, H., Luner, P., and Côté, W. (1982). "How retention aids change the distribution of filler in paper,” Tappi 65(4), 95-99.

Tarabara, V. V., Koyuncu, I., and Wiesner, M. R. (2004). "Effect of hydrodynamics and solution ionic strength on permeate flux in cross-flow filtration: Direct experimental observation of filter cake cross-sections,” J. Membrane Sci. 241(1), 65-78.

Thies-Weesie, D. M. E., and Philipse, A. P. (1994). "Liquid permeation of bidisperse colloidal hard-sphere packings and the Kozeny-Carman scaling relation,” J. Colloid Interface Sci. 162, 470-480. 
Tien, C., and Ramarao, B. V. (2008). Granular Filtration of Aerosols and Hydrosols, $2^{\text {nd }}$ Ed., Elsevier, Amsterdam.

Tien, C., Turian, R. M., and Pendse, H. P. (1979). "Simulation of the dynamic behavior of deep bed filters,” AIChE J. 25(3), 385-395.

Tobiason, J. E., and Vigneswaran, B. (1994). "Evaluation of a modified model for deep bed filtration,” Water Res. 28(2), 335-342.

Todd, A. C., Somerville, J. E., and Scott, G. (1984). “The application of depth of formation damage measurements in predicting water injectivity decline," SPE 12498, Denver, CO.

Tyner, J. S., Wright, W. C., and Lee, J. (2006). “Lagoon sealing and filter cakes,” Trans ASABE 49(2), 527-531.

Vaidya, R. N., and Fogler, H. S. (1990). "Formation damage due to colloidally induced fines migration,” Colloids Surf. 50, 215-229.

Veerpaneni, S., Wiesner, M. R. (1997). “Deposit morphology and head loss development in porous media,” Environ. Sci. Technol. 31(10), 2738-2744.

Vidyanathan, R., and Tien, C. (1987). "Hydrosol deposition in granular beds,” Chem. Eng. Sci. 43(2), 289-302.

Vigneswaran, S., and Chang, J. S. (1989). "Experimental testing of mathematical models describing the entire cycle of filtration,” Water Res. 23(11), 1413-1421.

Wang, Y. H., and Siu, W. K. (2006). "Structure characteristics and mechanical properties of kaolinite soils. I. Surface charges and structural characterizations," Can. Geotech. J. 43(6), 587-600.

Waterhouse, J. F., and Omori, K. (1993). "The effect of recycling on the fines contribution to selected paper properties,” in Products of Papermaking, Vol. 2, C. F. Baker, Ed., Pira Int'l., Leatherhead, UK, 1261-1292.

Wiesner, M. R. (1999). “Morphology of particle deposits,” J. Environ. Eng. 125(12), 1124-1132.

Wong, R. C. K., Ko, L. G., and Movassagh, R. (2004). “A study of flow tortuosity in locked oil sand,” J. Canadian Petroleum Technol. 43(1), 35-40.

Wu, Y. S., van Vlie, L. J., Frijlink, H. W., and Maarschalk, K. V. D. (2006). “The determination of relative path length as a measure for tortuosity in compacts using image analysis,” Eur. J. Pharm. Sci. 28(5), 433-440.

Yao, K. M., Habibian, M. M., and O’Melia, C. R. (1971). "Water and waste water filtration - Concepts and applications,” Environ. Sci. Technol. 5(11), 1105-1112.

Yeo, A. P. S., Law, A. W. K., and Fane, A. G. (2006). "Factors affecting the performance of a submerged hollow fiber bundle.” J. Membrane Sci. 280(1-2), 969-982.

Yoon, J. S., Culligan, P. J., and Germaine, J. T. (2006). "Visualization of particle behavior within a porous medium: Mechanisms for particle filtration and retardation during downward transport," Water Resource Res. 42(6), article no. W06417.

Whitney, R. P., Ingmanson, W. L., and Han, S. T. (1955). "Some aspects of permeation, filtration, and fluidization,” Tappi 38(3), 157-166.

Wildfong, V., Genco, J., Bousfield, D. W., and Hassler, J. (2003). “A twin wire drainage model for various blade configurations,” Proc. TAPPI Spring Technical Conf., 579590. 
Wildfong, V. J., Genco, J. M., Shands, J. A., and Bousfield, D. W. (2000a). "Filtration mechanics of sheet forming. Part I: Apparatus for determination of constant-pressure filtration resistance,” J. Pulp Paper Sci. 26(7), 250-254.

Wildfong, V. J., Genco, J. M., Shands, J. A., and Bousfield, D. W. (2000b). "Filtration mechanics of sheet forming. Part II: Influence of fine material and compression," $J$. Pulp Paper Sci. 26(8), 280-283.

Zeilinger, H., and Klein, M. (1995). "Modern measuring methods for cross-sectional filler distributions,” Wochenbl. Papierfabr. 123(20), 903-910.

Zhang, M., Li, C., Benjamin, M. M., and Chang, Y. (2003). "Fouling and natural organic matter removal in adsorbent/membrane systems for drinking water treatment," Environ. Sci. Technol. 37, 1663-1669.

Zhao, F., Landis, H. R., and Skerlos, S. J. (2005). "Modeling of porous filter permeability via image-based Stochastic reconstruction of spatial porosity correlations," Environ. Sci. Technol. 39(1), 239-247.

Zhao, F., Urbance, M., and Skerlos, S. J. (2004). "Mechanistic model of coaxial microfiltration for a semi-synthetic metalworking fluid microemulsion,” J. Manuf. Sci. Eng., Trans. ASME 126(3), 435-444.

Zhu, S., Pelton, R. H., and Collven, K. (1995). "Mechanistic modeling of fluid permeation through compressible fiber beds,” J. Chem. Eng. Sci. 50(22), 3557-3572. 